\title{
Experiments in the Coordinated Control of an Underwater Arm/ Vehicle System
}

Timothy W. McLain

Brigham Young University - Provo, mclain@byu.edu

Stephen M. Rock

Stanford University, rock@sun-valley.stanford.edu

Michael J. Lee

Monterey Bay Aquarium Research Institute

Follow this and additional works at: https://scholarsarchive.byu.edu/facpub

Part of the Mechanical Engineering Commons

\section{Original Publication Citation}

McLain, T., Rock, S., and Lee, M. Experiments in the Coordinated Control of an Underwater Arm/ Vehicle System, Journal of Autonomous Robots, 3, 213-232, 1996

\section{BYU ScholarsArchive Citation}

McLain, Timothy W.; Rock, Stephen M.; and Lee, Michael J., "Experiments in the Coordinated Control of an Underwater Arm/Vehicle System" (1996). Faculty Publications. 1522.

https://scholarsarchive.byu.edu/facpub/1522

This Peer-Reviewed Article is brought to you for free and open access by BYU ScholarsArchive. It has been accepted for inclusion in Faculty Publications by an authorized administrator of BYU ScholarsArchive. For more information, please contact ellen_amatangelo@byu.edu. 


\title{
Experiments in the Coordinated Control of an Underwater Arm/Vehicle System
}

\author{
Timothy W. McLain* Stephen M. Rock ${ }^{\dagger} \quad$ Michael J. Lee
}

\begin{abstract}
The addition of manipulators to small autonomous underwater vehicles (AUVs) can pose significant control challenges due to hydrodynamic interactions between the arm and the vehicle. Experiments conducted at the Monterey Bay Aquarium Research Institute (MBARI) using the OTTER vehicle have shown that dynamical interactions between an arm and a vehicle can be very significant. For the experiments reported in this paper, a single-link "arm" was mounted on OTTER. Tests showed that for 90-degree, two-second repetitive slews of the arm, the vehicle would move as much as 18 degrees in roll and 14 degrees in yaw when no vehicle control was applied.

Using a new, highly accurate model of the arm/vehicle hydrodynamic interaction forces, which was developed as part of this research, a coordinated arm/vehicle control strategy was implemented. Under this model-based approach, interaction forces acting on the vehicle due to arm motion were predicted and fed into the vehicle controller. Using this method, station-keeping capability was greatly enhanced. Errors at the manipulator end point were reduced by over a factor of six when compared to results when no control was applied to the vehicle and by a factor of 2.5 when compared to results from a standard independent arm and vehicle feedback control approach. Using the coordinated-control strategy, arm end-point settling times were reduced by a factor three when compared to those obtained with arm and vehicle feedback control alone. These dramatic performance improvements were obtained with only a five-percent increase in total applied thrust.
\end{abstract}

\section{Key Words and Phrases}

- underwater vehicle control

- underwater manipulator hydrodynamics

- underwater manipulator modeling

- underwater manipulator control

\footnotetext{
*Department of Mechanical Engineering, 242 CB, Brigham Young University, Provo, UT 84602, tmclain@et.byu.edu

†Department of Aeronautics and Astronautics, 250 Durand Building, Stanford University, Stanford, CA 94305, rock@sun-valley.stanford.edu

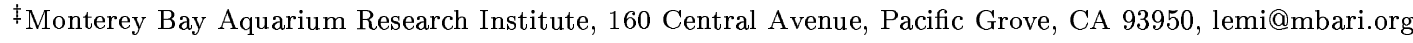


- coordinated control

- vehicle and manipulator control

- underwater vehicle experiments

\section{Introduction}

For users of remotely operated vehicles (ROVs), manipulators have become a valuable tool for performing a wide variety of tasks, from scientific sampling to maintenance and construction of underwater structures. Unlike ROVs, which tend to be quite stable statically, many smaller underwater vehicles have reduced static stability due to the small separation distance between their centers of mass and buoyancy. Also, for vehicles designed to travel efficiently through the water, the requirement for a small frontal area often limits the achievable static stability. The addition of manipulators to the vehicle makes control of the system more difficult because of the large hydrodynamic forces acting on the arm as it moves through the water. Hydrodynamic forces on the arm couple into the vehicle system, increasing the difficulty of regulating the position and attitude of the vehicle.

With the advent and implementation of higher levels of autonomy in the control of underwater robotic systems, it is becoming possible to move faster, and hence the relevance of hydrodynamic coupling is increased further. Today's advanced manipulator systems are typically tele-operated, using a passive "master" arm to control the underwater "slave" robot arm. These systems, are limited in a fundamental way by the skill, coordination, and endurance of the human operator. High-speed, precise motion is precluded by the limitations of the human/machine interface.

Human/machine interfaces incorporating increased autonomy, such as Task-Level Control [Wang et al., 1993], are capable of providing commands which exploit the full capabilities of manipulators for quick, precise motions. As the human operator is relieved of low-level control responsibilities, the limitations on system performance shift to the control systems implemented in place of the human. To enable high-performance control of a manipulator end point from a free-swimming vehicle base, low-level control systems that deal effectively with the complex hydrodynamics of fast motion must be developed. The development and implementation of such a controller is the focus of this paper.

A common work scenario for an underwater robot on a science mission is to perform a task, such as picking up a rock, sampling a biological specimen from the water column, or moving a science instrument, while holding the position and attitude of the vehicle fixed or "on station." Such tasks are examples of jobs where the station-keeping control of the vehicle is very important. By using control to keep the vehicle motionless, not only is the manipulation made easier, but the challenge of visually tracking the desired work scene is made less burdensome as well.

Station keeping is made difficult due to the presence of disturbances in the ocean environment such as those due to currents or tether forces. In the research presented here, the disturbance addressed is the large hydrodynamic coupling force generated as the arm moves through the water. If precise positioning of the manipulator end point is required, even relatively small motions at moderate speeds can have significant degrading effects. 
For the coordinated arm/vehicle control experiments discussed in this paper, a single-link arm was mounted on the OTTER vehicle, which is shown in Figure 1 and discussed in Section 3. Under the coordinated-control approach, the vehicle feedback controller was augmented with information about the hydrodynamic interaction forces between the arm and the vehicle. This information was produced using a very accurate model (developed during this work) of the hydrodynamic forces acting on a single-link arm. Although the single-link arm is quite simple mechanically, hydrodynamically it is quite complex due to the unsteady, three-dimensional flows developed as the arm moves. Although it does not possess the functionality of a full manipulator, the single link allows testing and validation

of the coordinated-control concept. It was necessary to develop a more advanced model because many of the unique hydrodynamic attributes of robotic motion (e.g., short unsteady swinging motions, radial-flow and tip-flow effects, etc.) had not been addressed by previous models. As hydrodynamic models for manipulators increase in sophistication to handle accurately multiple links and degrees of freedom, the control approach presented here can be extended easily to accommodate these systems.

The end-point-positioning and station-keeping performance of different controllers was tested by commanding the vehicle to maintain station while moving the arm from one position to another and then back to the original position. This point-to-point positioning task was chosen as the evaluation experiment for this research for two reasons. First, it is a generic task representative of other tasks of interest for an underwater system, such as sampling or pick-and-place maneuvers. Second, successful point-to-point positioning of the manipulator end point requires high-performance control of the entire arm/vehicle system. Such control is difficult to achieve without compensating for the hydrodynamic interaction forces explicitly in the control of the arm/vehicle system.

\section{Background}

Controlling underwater vehicles and robots to enable them to perform useful functions in the deep ocean represents a difficult problem that has challenged researchers for many years. Lead by the initial work of Yoerger and Slotine [Yoerger and Slotine, 1985], the application of sliding-mode control techniques to underwater vehicles became an active area of interest [Dougherty et al., 1988][Anderson, 1992][Healey and Lienard, 1993]. The motivation for using the sliding-mode approach is to enable robust control of the uncertain nonlinear vehicle system. Other research focused on using adaptive or neural-network control methods to deal with uncertainty in the plant model [Cristi et al., 1990][Goheen and Jefferys, 1990][Yuh, 1990][Yoerger and Slotine, 1991].

In the underwater-vehicle community, two theoretical efforts are of direct relevance to the coordinated arm/vehicle control approach taken in this research. In the work of Mahesh, Yuh, and Lakshmi [Mahesh et al., 1991], an adaptive controller for coordinating vehicle and arm motion was proposed. The arm and vehicle were considered as a single unit and an adaptive controller was developed for the whole system. This required a discrete-time approximation to the full nonlinear arm/vehicle dynamics to be implemented in the control. The success of the approach is dependent on the controller's ability to adapt accurately to rapidly changing hydrodynamic coefficients. The approach has been demonstrated using only a computer simulation of the planar motion of a vehicle. Experimental validation of the 
effectiveness of their approach has not been demonstrated.

In a second, related effort, Koval [Koval, 1994] proposed a model-based feedforward control approach for the stabilization of an underwater manipulation robot. In this work, the computational feasibility of a real-time hydrodynamic model implementation was addressed. Few implementation details were provided. No simulation or experimental results were given.

Several recent papers have addressed the modeling of underwater robotic systems [Lévesque and Richard, 1994] [McMillan et al., 1994][Tarn et al., 1995]. The focus of these papers is the efficient simulation of underwater vehicles and manipulators with many degrees of freedom. The models presented assume that drag and added-mass coefficients are constant. The latter two papers give an approach for calculating an estimate of the added-mass coefficient, but do not suggest a value for the drag coefficient. Lévesque does not include added-mass or other acceleration terms in his formulation, but suggests a value of 1.1 for the drag coefficient. None of these models were validated experimentally. Experimental results from this research have shown that for typical robotic motions, that the drag and added-mass coefficients of a swinging circular cylinder are not constant, but state-dependent functions of how far the cylinder has traveled.

Unlike the references presented here, the focus of this paper is not on increasing robustness or adapting to existing uncertainty, but rather on improving system performance by exploiting detailed knowledge of the system dynamics. The approach taken here involves augmenting the existing vehicle feedback control with information based on a fundamental physical understanding of the manipulator hydrodynamics, in a way that benefits the control of the

entire system. To achieve good results, this approach requires an accurate model of the manipulator hydrodynamics. In this paper, the coordinated-control approach is described and experimental validation is given.

\section{Experimental Apparatus}

The work presented here was performed as part of a joint research program between the Stanford University Aerospace Robotics Laboratory (ARL) and the Monterey Bay Aquarium Research Institute (MBARI). To enable experimental research in the ARL/MBARI program, a small underwater vehicle has been developed. OTTER (an Ocean Technologies Test-bed for Engineering Research) is described briefly below, while further detail can be found in [Wang et al., 1996].

For the arm/vehicle coordinated-control experiments presented in this paper, a single-link arm was mounted on the OTTER vehicle. Experiments were carried out in the MBARI test tank located in Moss Landing, California. The tank is $12 \mathrm{~m}$ in diameter and $4 \mathrm{~m}$ deep. The OTTER vehicle is about $2.1 \mathrm{~m}$ long, $0.95 \mathrm{~m}$ wide, and $0.45 \mathrm{~m}$ tall and weighs about $145 \mathrm{~kg}$ in air. A photograph of the vehicle with the arm mounted is shown in Figure 1. The main structural element of the vehicle is a $0.36 \mathrm{~m}$ diameter by $1.25 \mathrm{~m}$ long aluminum pressure housing which contains the on-board computers and sensors. Two $0.12 \mathrm{~m}$ diameter housings of the same length contain NiCad batteries that provide approximately $750 \mathrm{~W}$-h of power. The battery modules are mounted underneath the main housing.

The pressure housings are surrounded by eight ducted thrusters which provide propulsion to the vehicle. Each 
thruster housing contains its own commutation electronics and microcontroller that allow the motor to be current or velocity controlled. All components are mounted to a welded stainless-steel frame which surrounds the main housing and runs the length of the vehicle. The vehicle is covered by a streamlined fiberglass shell. Additional buoyancy is provided by fiberglass-covered redwood blocks stored within the shell. Because the shell is free flooding, the effective mass and inertia of the robot underwater are significantly higher than in air.

The arm used for the arm/vehicle control experiments was $7.1 \mathrm{~cm}$ in diameter and $1.0 \mathrm{~m}$ long. This length was chosen because it has roughly the same effective length as the prototype manipulator that has been designed for OTTER when it is in a nominal operating configuration. The arm was mounted from the fore-port corner of the vehicle frame and tilted down at an angle of 60 degrees from the horizontal (see Figure 1). This configuration was chosen because it places the arm in the region most likely to be the workspace of a future manipulator. With the arm mounted in this way, all of the vehicle degrees of freedom were affected by the forces generated as the arm moved.

In order to control the position and attitude of the vehicle, a variety of sensors were used. The horizontal $(x, y)$ position of the vehicle was measured using SHARPS, an acoustic long-baseline positioning system. The depth $(z)$ of the vehicle was sensed using a pressure transducer. Measurements of pitch and roll were provided by a dual-axis

inclinometer. Heading was measured using a flux-gate compass. Solid-state gyros were used to provide pitch, roll, and yaw angular rates. Each of these sensors is commercially available.

The computer hardware used for this research consisted of a UNIX-based Sun workstation networked to a VMEbased real-time computer system. The real-time computer hardware consisted of a two 68040-based single-board computers (on separate VME chassis) and a 16-channel, 12-bit analog input board housed on board the vehicle and a 68030-based single-board computer at the control station. One 68040 board was used for vehicle control, while the other was used for arm control. Communication to the arm motor was done at $31.25 \mathrm{kBaud}$ through a serial connection.

The real-time control software was developed in C++ for use under ControlShell [Rea, 1992], a software framework for real-time systems, which runs on top of the VxWorks operating system [Win, 1993].

\section{Approach}

\section{Dynamically Coordinated Control}

The central idea of dynamically coordinated control is to take advantage of physical understanding of system dynamics explicitly in the control of the arm/vehicle system. In the context of the control problem addressed here, this physical understanding is embodied in an accurate model of the manipulator hydrodynamic forces. Under the coordinatedcontrol approach, hydrodynamic and inertial forces generated from the motion of the arm are modeled in real time as the motion progresses. Based on the predicted interaction forces, thrust commands are sent to the thrusters to counteract the forces generated by the arm motion. In this way, the control of the arm and the vehicle are "coordinated." The experimental results presented in this paper demonstrate the validity of this coordinated-control approach for the arm and the vehicle. 
Figure 2 shows a simplified schematic diagram of the coordinated-control strategy. The main control components are the hydrodynamic model, the arm controller, the vehicle controller, the arm trajectory generator, and the vehicle trajectory generator. For the station-keeping experiments of this paper, the vehicle trajectory generator supplied zero-reference commands for each of the vehicle degrees of freedom. The control approach presented here was developed with the availability of an accurate hydrodynamic model in mind. The primary benefit of this modelbased control approach was the performance increase achieved by effectively eliminating one of the main disturbances on the system. Using the modeling approach presented here to predict the hydrodynamic forces has the additional benefit of maintaining high reliability and low cost — no additional sensors are required.

\section{Hydrodynamic Model}

The experimental validation of the coordinated-control approach of this paper was enabled, in part, by the development of a very accurate hydrodynamic model for the in-line forces ${ }^{1}$ acting on a circular cylinder rotating about its end. The theoretical foundation of this model is a two-dimensional analysis of the flow of an incompressible, inviscid fluid over a cylinder undergoing unsteady motions. The wake and feeding layers were modeled using discrete vortices with independent positions, velocities, and strengths. The analysis ignores the effects of skin drag, which are negligible for the manipulator motions considered. Figure 3 shows a schematic representation of the 2-D cylinder. The 2-D portion of this analysis is similar to that done by Sarpkaya [Sarpkaya, 1963, Sarpkaya and Garrison, 1963] for a stationary cylinder immersed in a moving fluid. Further details of the model can be found in [McLain, 1995].

The two-dimensional analysis resulted in the following equation for the acting hydrodynamic in-line force:

$$
F_{X}=C_{m}(s / D) \cdot \rho \frac{\pi D^{2}}{4} \frac{d U}{d t}+C_{d}(s / D) \cdot \frac{1}{2} \rho D U^{2} .
$$

The key outcome of this analysis was that for a cylinder undergoing constant acceleration motions, it was found that the state-dependent hydrodynamic drag and added-mass coefficients, $C_{d}$ and $C_{m}$, were functions of how far the cylinder had traveled only.

Using a standard strip-theory approach, the 2-D analysis was extended to three dimensions. This approach is diagrammed in Figure 4. The forces acting on a thin segment of the arm were calculated using a form of Equation 1:

$$
d F_{i}=C_{m_{i}}\left(s_{i} / D\right) \cdot \rho \frac{\pi D^{2}}{4} l_{i} d l_{i} \ddot{\theta}+C_{d_{i}}\left(s_{i} / D\right) \cdot \frac{1}{2} \rho D l_{i}^{2} d l_{i}|\dot{\theta}| \dot{\theta}
$$

The hydrodynamic in-line torque and force acting at the hub were found using the following simple relations:

$$
\begin{gathered}
d T_{i}=l_{i} d F_{i} \\
T_{\text {hyd }}=\sum_{i=1}^{n} d T_{i}
\end{gathered}
$$

\footnotetext{
${ }^{1}$ The net hydrodynamic force acting on the cross section of a cylinder is often viewed as the vector sum of the in-line forces and the transverse (lift) forces. In-line forces, which act in the plane of the cylinder's motion, are due to drag and added-mass effects. Transverse forces, which act normal to the plane of motion, are caused by the shedding of vortices into the wake.
} 


$$
F_{h y d}=\sum_{i=1}^{n} d F_{i}
$$

where $n$ was the number of segments used in the model.

Extensive measurements of forces and torques acting on the arm were used to identify the state-dependent behavior of $C_{d}$ and $C_{m}$. Flow visualization studies were also conducted to gain insight into the behavior of the flow and its effects on the forces acting. Figure 5 shows an example of the state-dependent drag and added-mass coefficients identified from the experimental data. In the initial stages of motion when the flow was attached, $C_{d}$ was 0 and $C_{m}$ was 1 , as potential-flow theory would indicate. As small symmetric vortices grew in the wake, $C_{d}$ increased and $C_{m}$ dropped off. When the symmetric vortex pair reached its maximum size, the values of $C_{d}$ and $C_{m}$ peaked. As the vortex wake became established, $C_{d}$ and $C_{m}$ settled in to quasi-steady-state values. Note that $C_{d}$ was significantly lower than the value of 1.2 that would be expected for a cylinder undergoing translational motion. This was due to three-dimensional flow effects induced by the rotational motion of a cylinder of finite length.

Figure 6 demonstrates the accuracy of the hydrodynamic model based on Equations 2 through 4 and Figure 5. It can be seen that for a wide range of motions, that the model accurately predicts the hydrodynamic torque acting. In addition to constant acceleration motions, the model worked equally well with fifth-order spline trajectories.

\section{Vehicle Feedback Control System}

For the fairly low speeds characteristic of station-keeping operation, the motion of the independent degrees of freedom of the vehicle are very lightly damped. This is due to the signed-quadratic relationship between velocity and fluid drag. At very low speeds, the drag is almost non-existent. At high speeds the drag forces are extremely large. For the low speeds and nominal horizontal configuration involved in station keeping, the $x, y$, and $z$ translational motions and the yaw motion can be modeled approximately as $1 / s^{2}$ plants, while the pitch and roll motions can be modeled as lightly damped second-order systems.

To provide control over the individual vehicle degrees of freedom, classical proportional-integral-derivative (PID) feedback controllers were used for each of the quantities $x, y, z, \phi, \theta$, and $\psi$. State-of-the-art position and attitude sensors provided the measurements required to achieve high-quality feedback control for each of the vehicle degrees of freedom. The integral portion of the control was implemented so that it was only active when the desired vehicle velocity was zero. In this way, good steady-state-error performance was achieved while preserving good transient response. Figure 7 shows a schematic representation of the vehicle feedback control implemented on the OTTER vehicle. The control loop was implemented digitally with a $100 \mathrm{~Hz}$ update rate. However, $x$ and $y$ position information from SHARPS was available at only $2.5 \mathrm{~Hz}$. Yaw information from the flux-gate compass was produced at $10 \mathrm{~Hz}$.

For station-keeping operations, the vehicle trajectory generator produces constant desired-position commands. These position commands are transformed into the vehicle-body frame (using the appropriate Euler-angle rotation matrix) for use by the controller. The vehicle controller takes in body-frame-referenced position and velocity measurements for the vehicle degrees of freedom and, based on the errors, produces a vector of three forces $\left(F_{c m d}^{x}, F_{c m d}^{y}, F_{c m d}^{z}\right)$ and three torques $\left(\tau_{c m d}^{x}, \tau_{c m d}^{y}, \tau_{c m d}^{z}\right)$ to be applied to the vehicle about its center of mass in the vehicle frame. Based 
on the thruster configuration, a vector of eight thrust commands $\left(T_{c m d}\right)$ for the vehicle can be calculated. This is done by recognizing that the $T_{c m d}$ required to produce the desired $F_{c m d}$ and $\tau_{c m d}$ can be calculated from the relation

$$
\left[\begin{array}{c}
F_{c m d} \\
\tau_{c m d}
\end{array}\right]=R_{v} T_{c m d}
$$

where $R_{v}$ is the $6 \times 8$ thruster matrix map that takes into account the position and orientation of the thrusters relative to the center of mass of the vehicle. Rearranging Equation 6 results in

$$
T_{c m d}=R_{v}^{\dagger}\left[\begin{array}{c}
F_{c m d} \\
\tau_{c m d}
\end{array}\right]
$$

where $R_{v}^{\dagger}$ is the pseudo-inverse $\mathrm{e}^{2}$ of $R_{v}$. Since the configuration of the thrusters on the vehicle is known a priori, $R_{v}$ and $R_{v}^{\dagger}$ can be computed beforehand without impacting the speed of the real-time implementation.

Past research has shown that good agreement between the commanded thrust, $T_{c m d}$ and the actual thrust produced by the thrusters can be achieved by controlling the thruster motors in velocity mode and taking advantage of the relationship between thrust produced and the angular velocity of the output shaft squared [Healey et al., 1995]. It has been found that for the steady-thrust case that

$$
T=k_{\omega} \omega|\omega|
$$

In other words, the angular velocity command required to produce a desired thrust command can be calculated from

$$
\omega_{c m d}=k_{T} \operatorname{sgn}\left(T_{c m d}\right) \sqrt{\left|T_{c m d}\right|} .
$$

Values of $k_{T}$ for each of the thrusters were determined from thruster-velocity measurements together with spring-scale measurements of thrust on the vehicle. From Equation 9, thruster angular velocity commands can be determined for each of the thrusters. These commands are sent to the thrusters, causing the vehicle to move in the desired way.

Achievable feedback gains were limited in two ways. First, low update rates on $x, y$ and yaw limited the amount of proportional feedback that could be applied without causing an instability. In $x$ and $y$, derivative information was available only from differencing the $2.5 \mathrm{~Hz}$ SHARPS measurement, so it was difficult to get the good velocity information needed for high derivative gains. Second, noise on the sensors (particularly yaw) resulted in very noisy control signals which caused excessive damage and wear to thrusters when high gains were used. These signals could have been be filtered more, but this would have resulted in more phase delay which would have had a destabilizing effect. Even with these sensor limitations, in the absence of arm motion, good positioning performance was achieved using PID control on the individual degrees of freedom of the vehicle.

\footnotetext{
${ }^{2}$ using the Moore-Penrose pseudo-inverse, $A^{\dagger}=A^{T}\left(A A^{T}\right)^{-1}$
} 


\section{Arm Feedback Control System}

For the single link, the in-line hydrodynamic forces, though nonlinear and somewhat uncertain, provided damping and stability to the arm dynamics. Because of the well-damped dynamic characteristics of the arm, high position feedback gains were achievable. Using straightforward implementation of classical control methods, very good position control was attained.

Figure 8 shows a schematic block diagram of the arm controller implemented for the experiments described in this paper. This implementation takes advantage of the $1 \mathrm{kHz}$, high-gain velocity feedback controller internal to the motor electronics. By controlling the arm motor in "velocity control" mode, the arm actuator behaved as a velocity source.

A fifth-order trajectory generator was used to provide smooth desired commands to the controller. Desired velocity commands direct from the trajectory generator were sent to the motor as feedforward signals. A proportional position feedback loop was closed around the internal velocity feedback loop to provide control of the arm joint angle $\alpha$. The sample rate was limited to $230 \mathrm{~Hz}$ by the achievable serial communication bandwidth between the VME cage and the $68 \mathrm{HC} 11$ microcontroller in the motor housing.

\section{Coordinated-Control Implementation}

The coordinated-control approach implemented here involved augmenting existing independent arm and vehicle controllers with information about their dynamic interaction to produce improved control of the system. Figure 9 shows a schematic representation of the coordinated-control approach implemented on the OTTER system. The coordinating information between the two systems came from the manipulator hydrodynamic model and the decoupling thrust commands it generated.

\section{Implementation Assumptions}

The essential pieces of information necessary for implementation of the single-link manipulator hydrodynamic model developed and described above are the position, velocity, and acceleration of the link relative to the water. In implementing this approach, four key assumptions were made:

1. Vehicle motions are small and do not contribute significantly to the net motion of the manipulator relative to the water.

2. Desired arm-joint acceleration is a good approximation of the actual joint acceleration.

3. The water through which the arm is moving is still.

4. Transverse (lift) forces are insignificant in comparison to the in-line forces.

Under the condition that the vehicle and arm controllers are functioning as intended, the first two assumptions are reasonable and valid. The benefit of the first assumption is an increase in the control bandwidth due both to 
a reduction in computational complexity and to a decrease in the amount of information passed over the limitedbandwidth communication link between the arm and the vehicle. The benefit of the second assumption (which is standard for many model-based robot controllers) is that measurements of individual joint accelerations are not required. The third assumption is valid for the tests reported here: In the large MBARI tank, fluid motion is due solely to thruster discharge which does not impinge on the arm. The validity of the fourth assumption has been demonstrated experimentally at the ARL [McLain, 1995]. For the apparatus used and the types of motions considered here, strong periodic vortex shedding did not become well-established, hence transverse (lift) forces are comparatively very small.

Figure 10 shows the hydrodynamic model performance under the assumptions outlined above. The agreement between the measured arm-joint torque and the modeled arm-joint torque was quite good. Some errors existed at the beginning and end of arm trajectories due to accelerations, caused by joint flexibility, which were not incorporated into the model when the desired acceleration signal was used. Errors during the middle of the trajectory (near the torque peaks) were due primarily to vehicle motions which were not included in the model. In spite of the simplifying assumptions made, it can be seen that good modeling accuracy was maintained.

\section{Implementation Description}

Feedback controllers were applied to the arm and vehicle as described above. In addition, the hydrodynamic model and decoupling control connection were added to complete the coordinated-control implementation as shown in Figure 9. To provide the rate information required for the model, the arm position signal was pseudo-differentiated using a digital filter. Position information came directly from the motor encoder, while the desired acceleration signal was used to provide the required acceleration information.

The hydrodynamic model was implemented as described above. The output of the model was a vector of three forces $\left(F_{h y d}^{x}, F_{h y d}^{y}, F_{h y d}^{z}\right)$ and three torques $\left(\tau_{h y d}^{x}, \tau_{h y d}^{y}, \tau_{h y d}^{z}\right)$ acting about the base of the arm joint. These were the forces and torques required to counteract the forces generated by the motion of the arm. As with the feedback control, a thruster configuration map was used to determine the required decoupling control commands to the thrusters, $T_{d c p l}$, to counteract the hydrodynamic coupling forces:

$$
\begin{aligned}
& {\left[\begin{array}{c}
F_{h y d} \\
\tau_{h y d}
\end{array}\right]=R_{a} T_{d c p l} .} \\
& T_{d c p l}=R_{a}^{\dagger}\left[\begin{array}{c}
F_{h y d} \\
\tau_{h y d}
\end{array}\right]
\end{aligned}
$$

$R_{a}$ was the $6 \times 8$ thruster matrix map that took into account the position and orientation of the thrusters relative to the base of the arm.

The vector $T_{d c p l}$ of decoupling thruster commands was sent to the vehicle controller 60 times per second over an Ethernet connection between the arm and vehicle card cages. These decoupling commands were summed directly with the feedback commands, $T_{f b a c k}$, to produce the the total thrust command to be sent to the thrusters, $T_{c m d}$. 
As shown in Figure 9, the model-based decoupling control implemented here is calculated based upon the desired arm acceleration and upon measurements of arm position and velocity. As such, the output of the decoupling controller is a combination of nonlinear, model-based feedforward and feedback control (though not error based). This nonlinear feedforward and feedback combination is used to "cancel" the undesirable nonlinear coupling between two components of the system. In this regard, it is similar to computed-torque control [Craig, 1989], which uses model-based feedback and feedforward loops to linearize and decouple the controlled system.

Combining feedback control with decoupling control, as depicted in Figure 9, results in a vehicle controller that possesses the positive attributes of both types of control. Feedback control provides regulation capability, robustness to disturbances, and robustness to plant model uncertainties. However, feedback control is inherently error-based. This implies that an error must exist before the controller does anything in response. In this situation, a predictive model providing decoupling control commands is very useful. Rather than waiting for an error to build up, decoupling control predicts what the control command should be to regulate the errors in the system before the errors occur.

The limitation of the decoupling control implemented here is that since it was designed to cancel a particular interaction, it (alone) does not reject errors due to unknown disturbances or uncertainty in the plant model: Its nonlinear feedforward and feedback loops are only active when the arm moves, and even when active they do not provide the position and attitude regulation capability required for precise control of an underwater vehicle system. For the problem considered here, a balanced combination of decoupling control and position feedback control provides the best solution.

\section{Experimental Test Strategy}

To determine the value of the proposed coordinated-control strategy, four different vehicle controllers were implemented and tested. In each of the four evaluated vehicle controllers, the arm control used was identical (see Figure 8). The four different controllers evaluated are briefly described below.

No Vehicle Control In this case, both the decoupling path from the arm hydrodynamic model and the vehicle feedback control loop were open. With no control active, the effects of arm motion on the open-loop vehicle dynamics were observed.

Feedback Control Only In this control configuration, the vehicle feedback control loop was closed while the decoupling path from the arm model to the vehicle remained open. In this case, the effects of arm motion on the closed-loop vehicle dynamics were seen and the disturbance rejection capabilities of closed-loop control were demonstrated.

Decoupling Control Only In this implementation, the vehicle feedback control loop remained open while the decoupling path from the hydrodynamic model to the vehicle was closed. Using this configuration, the effectiveness and accuracy of the decoupling control application were determined. 
Feedback with Decoupling Control In this case, both the vehicle feedback control loop and the decoupling path from the arm model were closed. In this control configuration, the performance benefits of combining the decoupling control, which provides predictive coordination between the motion of the arm and control of the vehicle, with the vehicle feedback control, which provides robustness to disturbances and uncertainty, were tested.

The Feedback Control Only test case, along with the No Vehicle Control case, provide performance baselines against which the Feedback with Decoupling Control approach are compared.

\section{$5 \quad$ Experimental Results}

This section presents results from the coordinated arm/vehicle control experiments. Data from two different types of tests are presented - multiple-swing motions and single-swing motions of the arm. In Figures 11 through 18, data from experiments where the arm was swung back and forth between two positions multiple times are shown. In Figures 19 through 21, data are presented where the arm was slewed a single time from one point to another.

Several different types of data are presented including image sequences from video footage of the experiments, vehicle error regulation data, arm end-point error data, and thruster usage data. Using these results comparisons are drawn between the different controller types. The results demonstrate the benefits of both feedback control and decoupling control and their complementary attributes that result in the best control behavior when feedback and decoupling control are combined.

\section{Vehicle Station Keeping}

Figures 12 through 15 show image sequences taken from video footage shot during arm/vehicle control experiments with the OTTER vehicle. In each sequence, the images were taken at approximately one-second intervals during the first three swings of the arm in a multiple-swing sequence. Figure 11 shows a typical time history of the arm joint angle for the multiple-swing motions of the arm. The image sequences give a qualitative feel for the performance of the different controllers.

No Vehicle Control Figure 12 shows images taken for the No Vehicle Control case. It can be seen that the open-loop roll mode of the vehicle was excited by the motion of the arm. Errors in roll were as large as 18 degrees in both directions from the horizontal. A significant error in yaw can also be observed. During this sequence, the vehicle drifted about 15 degrees in yaw from its initial heading. These images demonstrate that the hydrodynamic coupling forces involved in moving an arm at moderately fast speeds are very large and that they have a significant degrading effect on the station-keeping capability of a small, agile vehicle such as OTTER.

Feedback Control Only When compared with the No Vehicle Control case, the benefits of feedback control, as shown in the sequences of Figure 13, are readily apparent. Errors in yaw and roll were reduced, but still very significant. The closed-loop roll mode, although much more damped than the open-loop mode, was still excited by 
the arm motion. Roll motions were as large as nine degrees in both directions. The yaw angle of the vehicle varied as much as eight degrees from its nominal position. While the benefits of closed-loop control are obvious from this sequence of images, the disturbances introduced from the arm/vehicle coupling still resulted in substantial deviations in the vehicle's position and attitude.

Decoupling Control Only The sequence of Figure 14 illustrates the performance of the controller in the Decoupling Control case. It can be seen that the influence of the arm motion on the vehicle was greatly reduced. Errors in roll and yaw were noticeably smaller. Since the application of the decoupling control did not perfectly cancel the interaction forces, the open-loop roll mode was excited slightly by the combination of arm and thruster forces acting.

Feedback with Decoupling Control Figure 15 shows the performance results obtained using the Feedback with Decoupling Control approach. As with the Decoupling Control Only case, the hydrodynamic interaction forces were largely canceled by the decoupling component of the control. The advantage of adding the feedback control was that the remaining errors from the inexact decoupling control were further reduced by the error regulation of the feedback control. With the addition of feedback control, robustness to system uncertainty was provided and the tendency of the vehicle to drift off station was eliminated. These benefits are demonstrated clearly in a more quantitative way in the following sections.

As a final comparison of the station-keeping performance of the different vehicle controllers, selected vehicle roll and yaw angle data are presented. In this particular application, errors in roll and yaw were the largest contributors to the arm end-point error, especially in the cases of No Vehicle Control and Feedback Control Only.

Vehicle Roll Error Figure 16 shows time histories of vehicle roll error data for each of the four controller types considered. With no control effort, roll errors were very large (between \pm 18 degrees) as the open-loop roll mode of the vehicle was excited. The addition of feedback control improved the roll error regulating performance, but the errors were still significant (between \pm 9 degrees). Decoupling control alone effectively countered much of the roll moment generated from the arm motion. In this case, decoupling control allowed the vehicle to respond to arm interaction forces before significant attitude errors were induced. Further improvement was realized when decoupling and feedback control were combined. Peak roll errors were limited to less than 1.5 degrees in this case.

Vehicle Yaw Error Time histories of vehicle yaw error for the different controllers are shown in Figure 17. When no feedback or decoupling control was applied, the vehicle heading angle drifted significantly from its nominal position. Unlike the roll and pitch attitude degrees of freedom, the yaw degree of freedom has no passive restoring force inherent to its open-loop dynamics. Because of this the yaw degree of freedom was fully dependent on feedback control to prevent drifting due to disturbances or uncertainty in the plant model. When feedback control alone was applied, the tendency to drift was reduced, but the closed-loop dynamics of the yaw controller became apparent. As the controller attempted to reject the yaw disturbance, it caused the vehicle to oscillate significantly in response (up to 9 degrees error). When decoupling control only was applied, the yaw disturbance due to arm motion effectively was 
canceled resulting in much smaller yaw errors. With feedback and decoupling control combined, the yaw errors were again small. Yaw errors were roughly three times smaller for the Decoupling Only and Feedback with Decoupling cases than for the Feedback Only case.

Summary For each of the degrees of freedom of the vehicle, the best error regulation results were obtained when both decoupling and feedback control were combined. The decoupling control effectively canceled most of the dynamical coupling between the arm and the vehicle. Much of the remaining coupling effect was eliminated by the feedback control. The feedback control also provided for the rejection of other disturbances (e.g., tether forces, currents) and robustness to uncertainties in the plant.

\section{Arm End-Point Positioning Performance}

While not sensed and controlled directly in these experiments, arm end-point position error is a useful indicator of the quality of the performance of the arm/vehicle controller. End-point position data were generated by post-processing vehicle position and attitude and arm position measurements based on the kinematic configuration of the system. Data sampling on the vehicle controller and arm controller was synchronously triggered to allow correlation of arm and vehicle data in time.

For the multiple-swing motions of the arm, Figure 18 shows time histories of the arm end-point error for the four controllers tested. The mean end-point errors were calculated to be $28 \mathrm{~cm}$ for the No Vehicle Control case, $11 \mathrm{~cm}$ for the Feedback Control Only case, $9.1 \mathrm{~cm}$ for the Decoupling Control Only case, and $4.6 \mathrm{~cm}$ for the Feedback with Decoupling Control case. It can be seen that with combined decoupling and feedback control, that the end-point errors were reduced by a factor of six when compared with the No Vehicle Control case and a factor of 2.5 when compared to the Feedback Control Only case. In the Feedback with Decoupling Control case, where end-point errors were smallest, a more significant portion of the error can be attributed to the arm joint-angle error. During the arm slews, joint tracking errors were typically around 2 to $3 \mathrm{~cm}$.

\section{Arm End-Point Settling-Time Performance}

Figure 19 shows plots of the distance of the arm end point from the desired target for the four different controllers. This data was obtained by slewing the arm through 90 degrees in two seconds. For the slews considered, the distance traveled by the end point was about $1.5 \mathrm{~m}$. Here settling time is defined as the time required to stay within five percent (of the total distance traveled) of the target - in this case $\pm 7.5 \mathrm{~cm}$.

These settling-time plots demonstrate the importance of the feedback component of the vehicle control. Without feedback, the arm end point either fails to come within five percent of the target (as in the No Vehicle Control case) or it fails to remain within the five-percent error bound around the target point (as in the Decoupling Control Only case).

In the Feedback Control Only case, the time required to settle to within five percent of the target was about 6.5 seconds. As the arm moved, significant errors in roll, yaw, $x$, and $y$ resulted. Coming and staying within the error 
bound required these errors to be reduced which took a substantial amount of time.

In the Feedback with Decoupling Control case, the observed settling time was about two seconds. This represents an improvement of over three times compared to the settling time of the Feedback Control case. Because the vehicle stayed on station, the settling time corresponded directly to the duration of the slew.

\section{Thruster Usage}

Horizontal Thrusters Figure 20 shows thruster responses for each of the horizontal thrusters on the vehicle for the Feedback Only and Feedback with Decoupling cases. For each thruster, it can be seen that the Feedback with Decoupling thrusts spiked up to comparatively large values during the slew, but then settled to very small values almost immediately after the slew ended. On the other hand, for the Feedback Only case it can be seen that the thruster commands were much smaller initially, but that the thrusters fired over much longer durations. Long after the thrusters had essentially shut off in the Feedback with Decoupling case, they continued to fire in the Feedback Only case in an effort to regulate the errors in the system. These plots illustrate the predictive capability of the decoupling control, which in each case leads the feedback control in responding to the arm motion.

Note that the largest thrust command by far came from the lateral-fore thruster. This makes sense physically since the lateral-fore thruster was positioned very close to the base of the arm joint and the lateral forces generated by the arm were very large. The responsibility for countering these forces fell largely upon the lateral-fore thruster.

Vertical Thrusters Figure 21 shows thruster responses for each of the vertical thrusters on the vehicle for the Feedback Only and Feedback with Decoupling cases. As with the horizontal thrusters, the vertical Feedback with Decoupling thrust commands spiked up to large values during the arm motion to compensate for the interaction forces generated. Unlike the horizontal thrusters, the vertical thrusters responded fairly quickly to the roll errors introduced in the Feedback Only case. Notice that in both control cases the vertical-aft thrusters remained on well after the completion of the arm motion. This was due to the integral control acting to zero the pitch error of the vehicle.

Comparison of Thruster Usage Two useful indicators of thruster usage are peak thrust applied and the total thrust $^{3}$ applied over the 20-second duration shown. Peak thrusts give a good indication of worst-case instantaneous demand on the thrusters, while total thrust usage gives a better indication the total thrust (and hence battery power) required over the duration of the test. For the thruster data shown in Figures 20 and 21, the peak thrusts were 1.8 to 2.7 times larger in the Feedback with Decoupling case, with the exception of the lateral-fore thruster where it was five times larger. While the peaks are much higher due to the decoupling component of the command coming from the hydrodynamic model, the thrust durations were shorter for the Feedback with Decoupling case. This is reflected in the relative values of the total thrust used in each control case: Only five percent more total thrust was used in the Feedback with Decoupling case than for the Feedback Only case.

\footnotetext{
${ }^{3}$ Total thrust was calculated by summing the absolute values of the thrusts applied over the duration of the test, while subtracting out any DC components. This is analogous to evaluating the expression $\int\left|T_{c m d}\right| d t$.
} 
The key result of this comparison is that for a slight increase in total thrust used, a very substantial performance improvement was realized (see Figure 19). This was accomplished by taking advantage of knowledge of the system dynamics and coordinating the control in a sensible way.

\section{Conclusions}

In this paper, an intuitively straightforward approach developed for the coordinated control of an underwater arm/vehicle system has been described in detail. Relevant background information has been presented along with detailed discussion of the control system implementation. Experiments demonstrated that the hydrodynamic coupling forces between an arm and a vehicle can be very significant, resulting in large disturbances to the station-keeping control of the vehicle. Experimental results showed that substantial performance improvements can be realized in the control of an underwater arm/vehicle system by incorporating model-based information about the hydrodynamic coupling into the control of the system. Tracking errors at the manipulator end point were typically reduced by over a factor of six using the coordinated control approach when compared to the vehicle with no control applied and by a factor of 2.5 when compared to the standard approach of independent arm and vehicle feedback controllers. Using the approach presented here, settling times at the manipulator end point were also reduced by over three times when compared to the values obtained using feedback control alone. It is important to note that these dramatic performance improvements were achieved with only a five-percent increase in total control effort.

\section{Acknowledgments}

This research was supported by the Monterey Bay Aquarium Research Institute, ONR Contract N0014-92-J-1943, and NASA Contract NCC 2-333. This research has benefited greatly from the contributions of the other participants in the ARL/MBARI research program: Dick Burton, Howard Wang, Rick Marks, David Miles, Glen Sapilewski, Kortney Leabourne, and Steve Fleischer. 


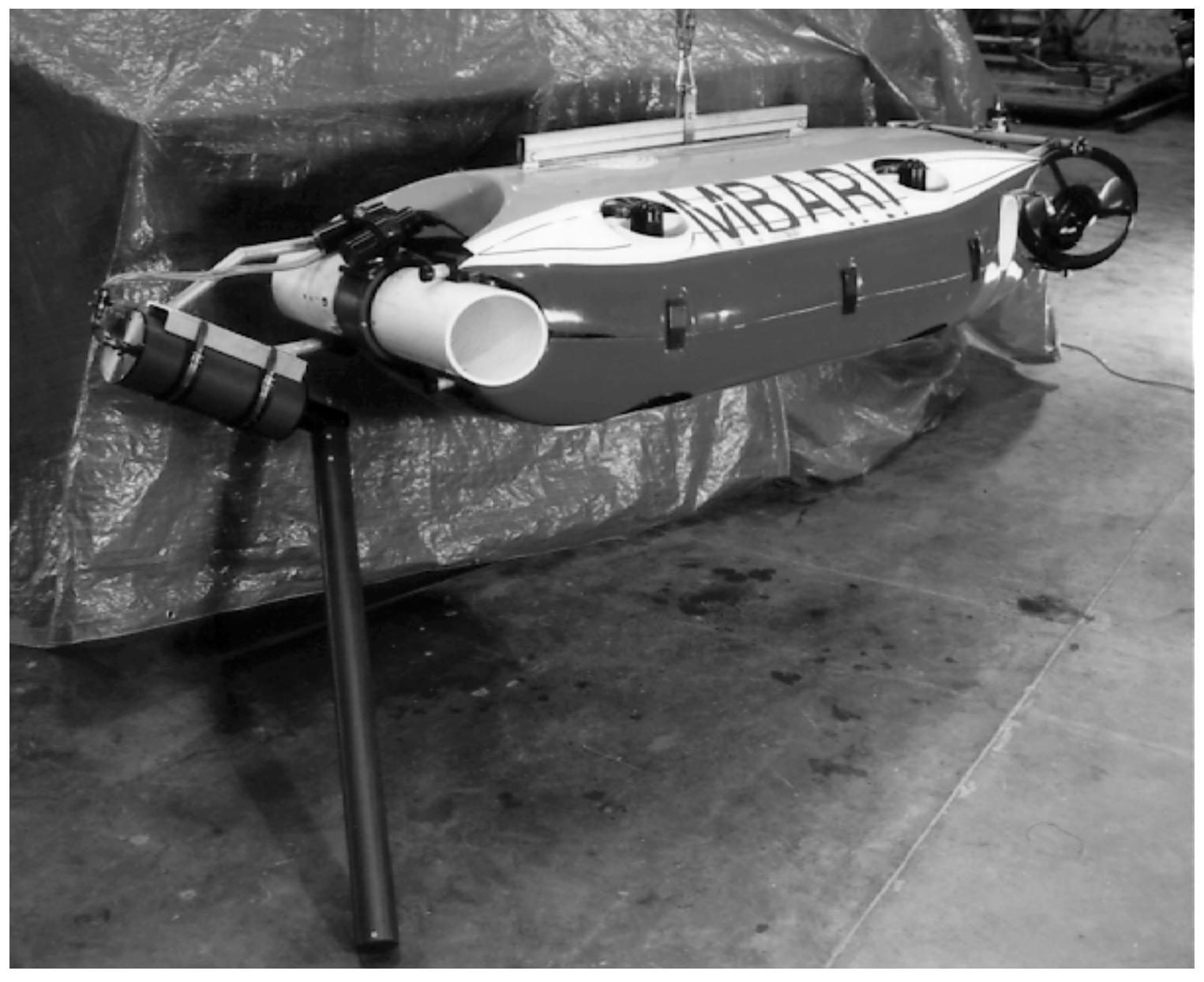

Figure 1: OTTER Vehicle With Single-Link Arm

This photo shows the OTTER vehicle with the single-link manipulator mounted. The single-link manipulator is $7.1 \mathrm{~cm}$ in diameter and $1.0 \mathrm{~m}$ long, while the vehicle is $2.1 \mathrm{~m}$ long, $0.95 \mathrm{~m}$ wide and $0.45 \mathrm{~m}$ tall. 


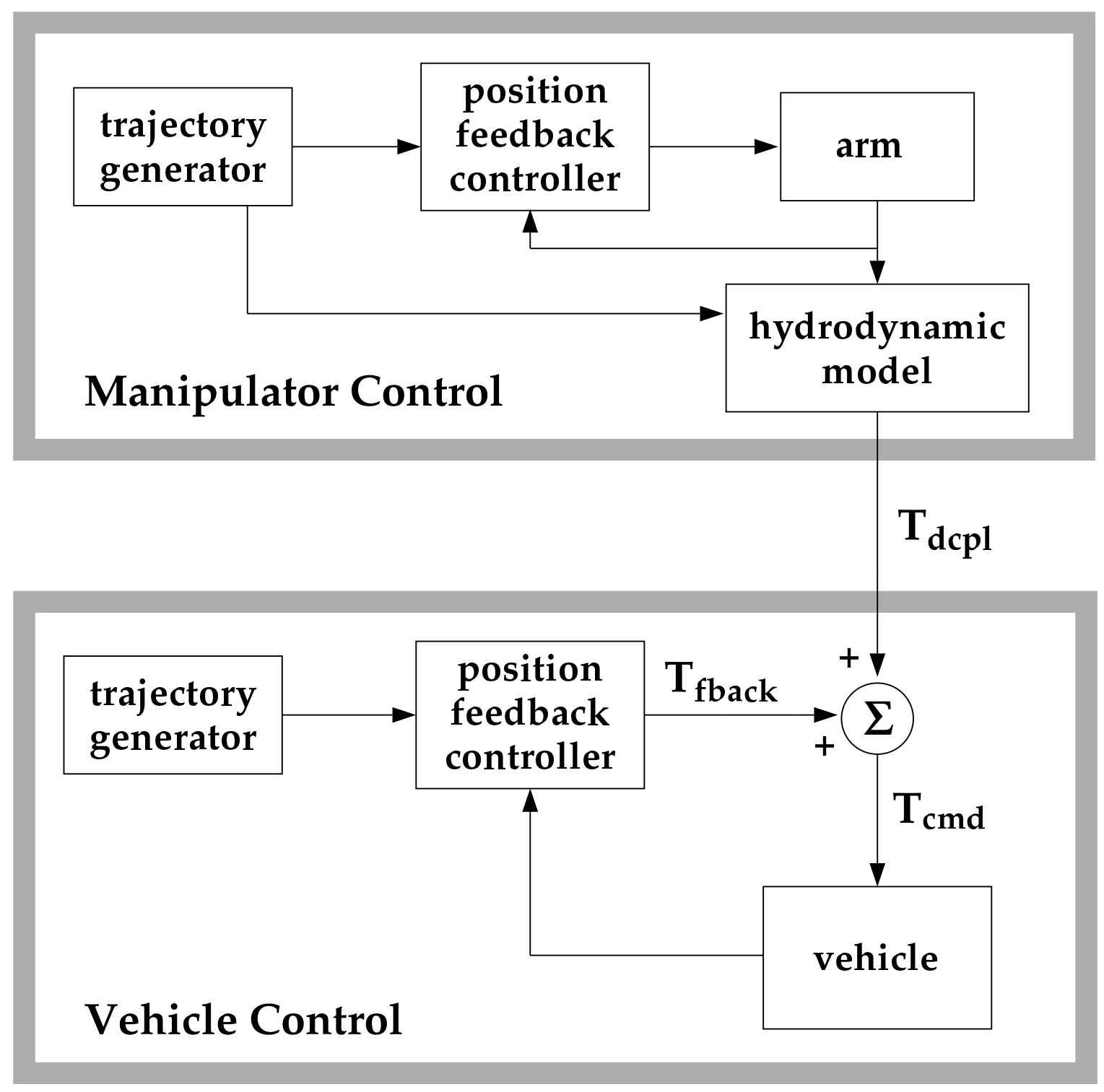

Figure 2: Block Diagram of Primary Coordinated-Control Components

This schematic shows a simplified block diagram for the coordinated-control approach. The primary components are the hydrodynamic model, the manipulator and vehicle controllers, and the manipulator and vehicle trajectory generators. 


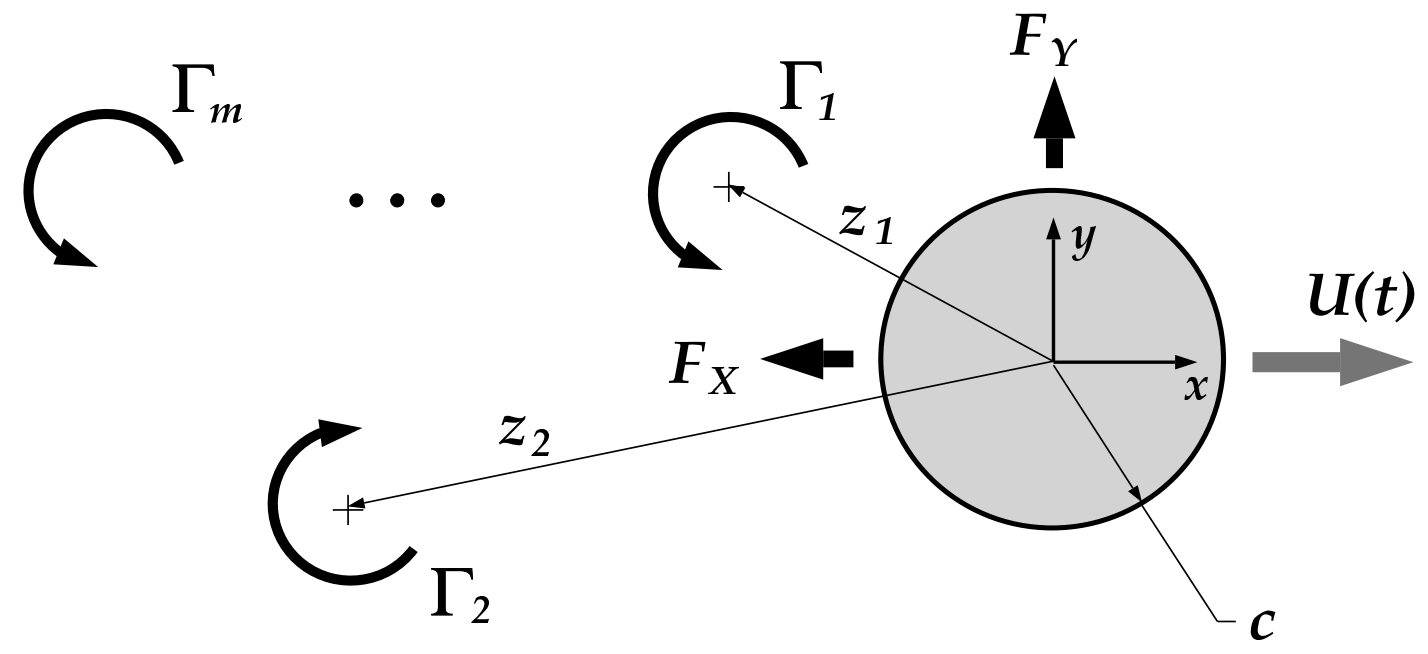

Figure 3: Two-Dimensional Hydrodynamic Model Schematic

This diagram illustrates the flow conditions modeled in the 2-D hydrodynamic analysis. 


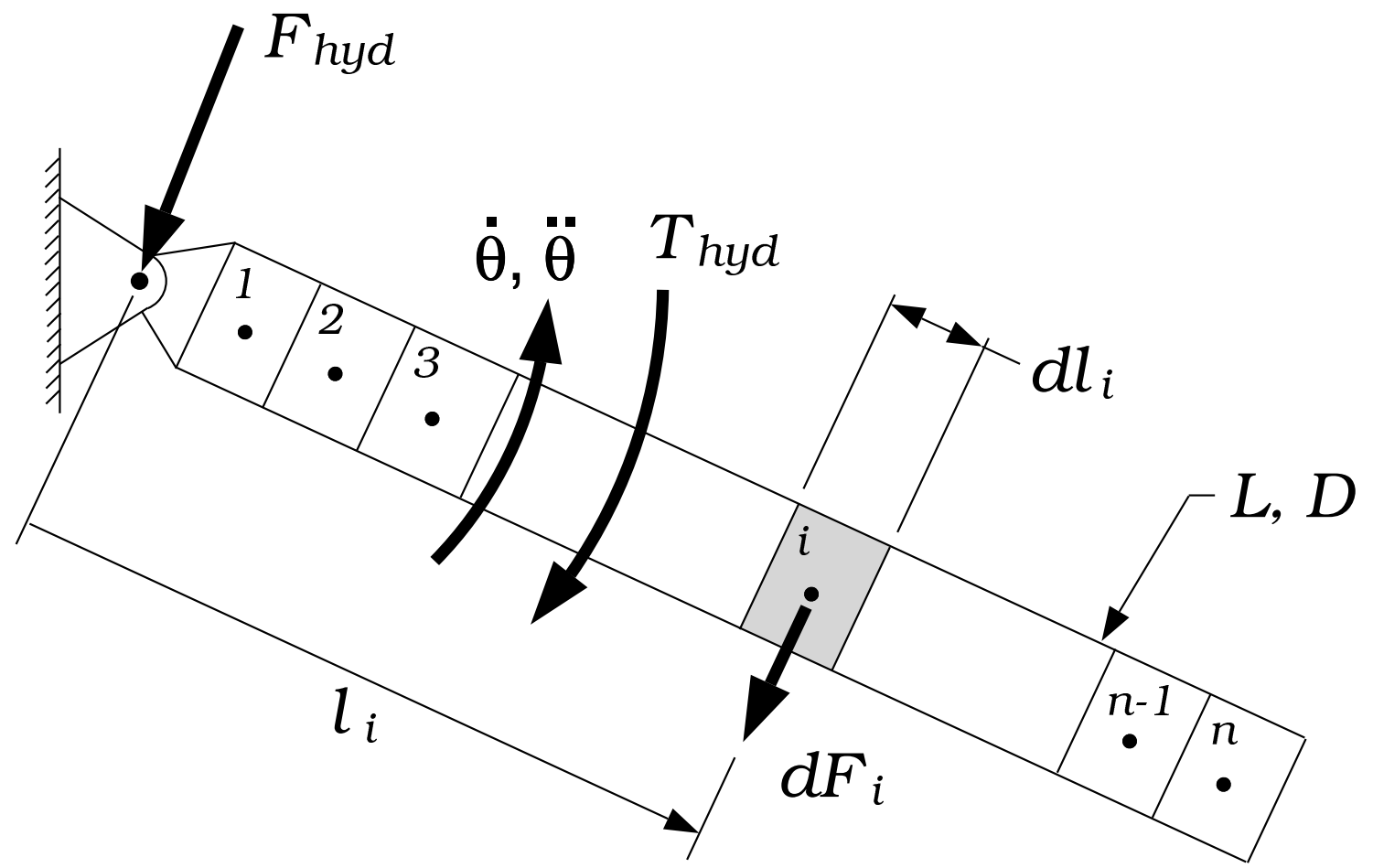

Figure 4: Diagram of Strip-Theory Implementation

This figure shows how strip-theory was applied to extend the 2-D hydrodynamic analysis to 3-D. 

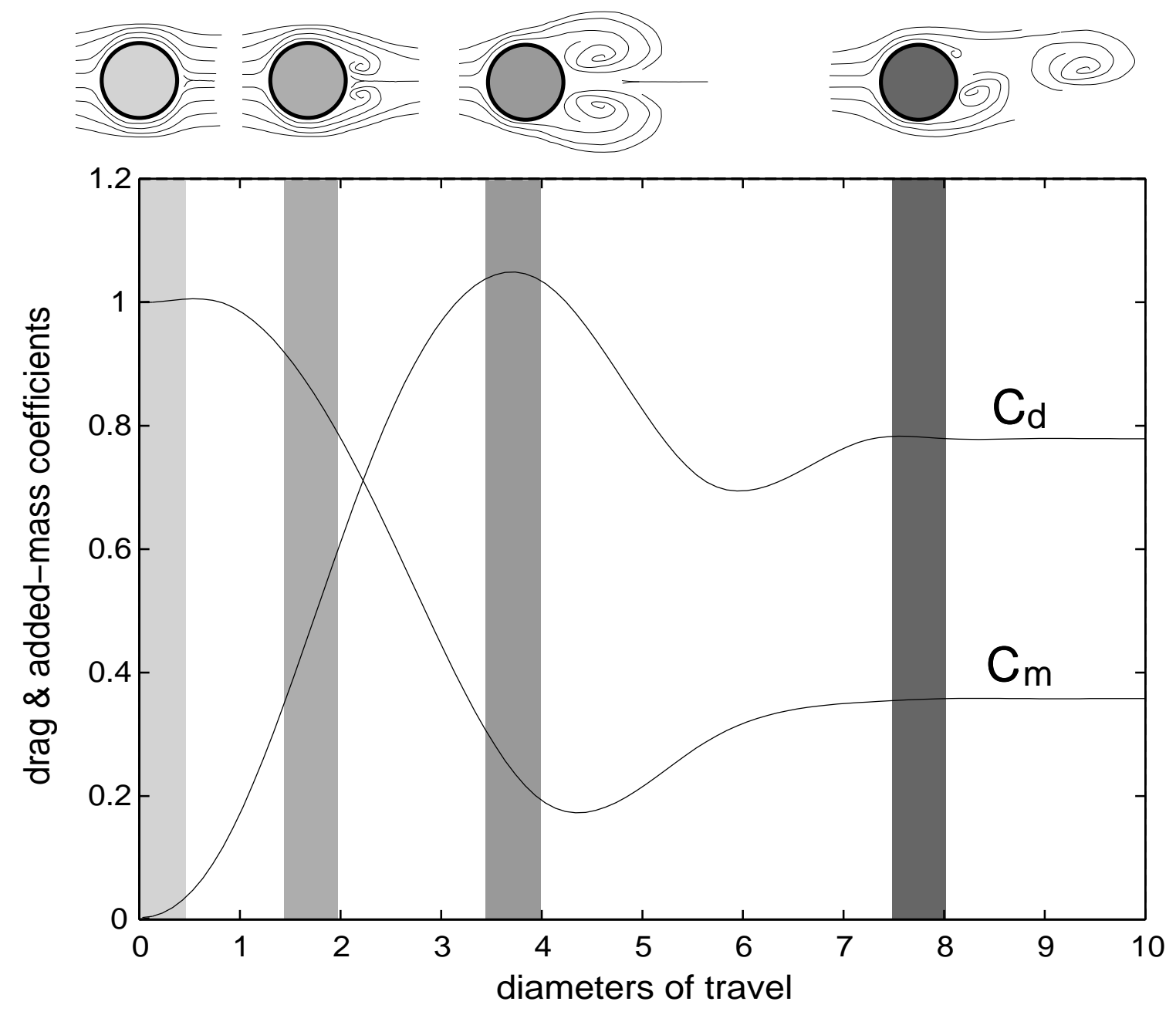

Figure 5: State-Dependent Drag and Added-Mass Coefficients

This plot shows an example of the behavior of drag and added-mass coefficients as functions of travel for a swinging circular cylinder. 

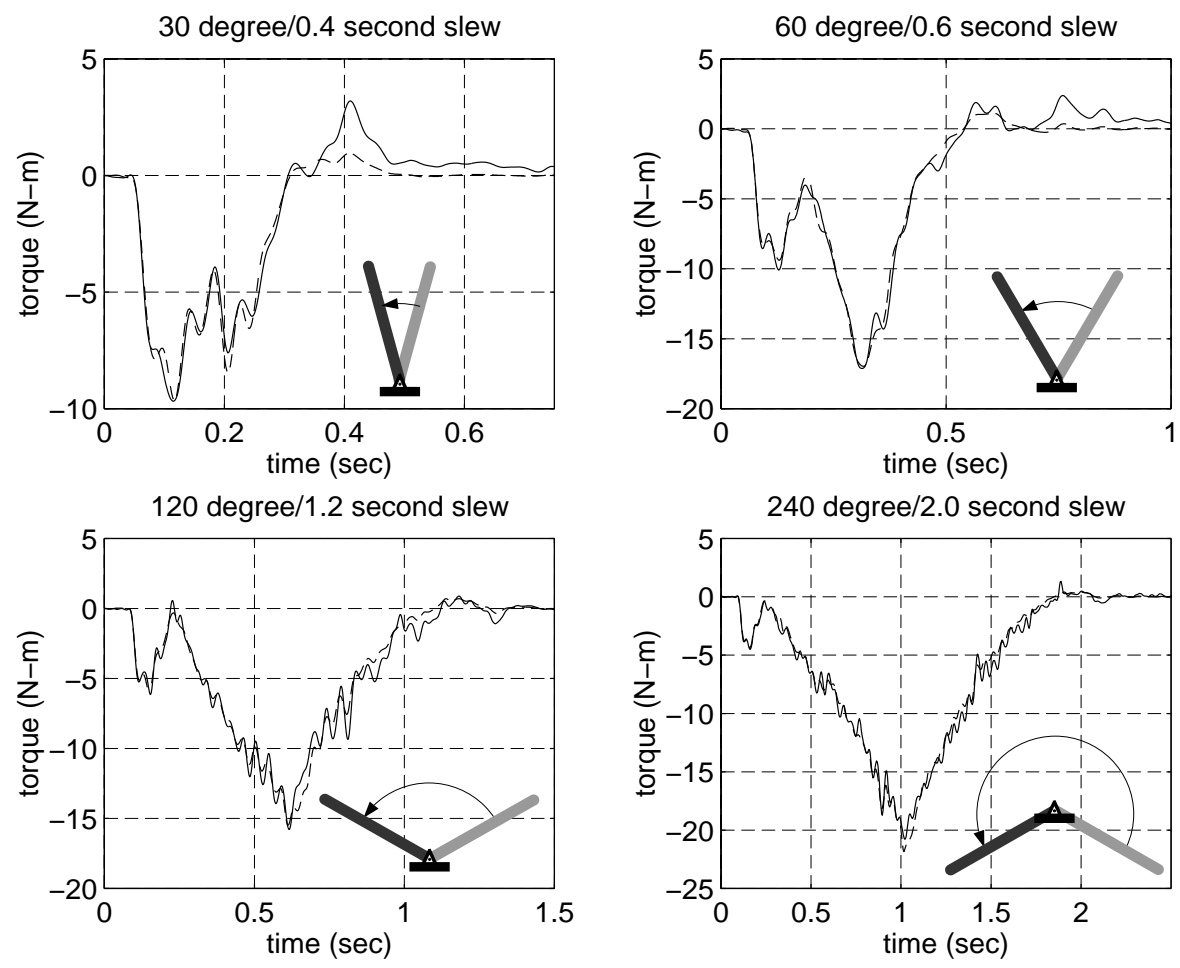

Figure 6: Experimental Hydrodynamic Modeling Results

Hydrodynamic torque model results are plotted for 30-degree, 60-degree, 120-degree, and 240-degree motions. The hydrodynamic model is accurate over a wide range of motions. 


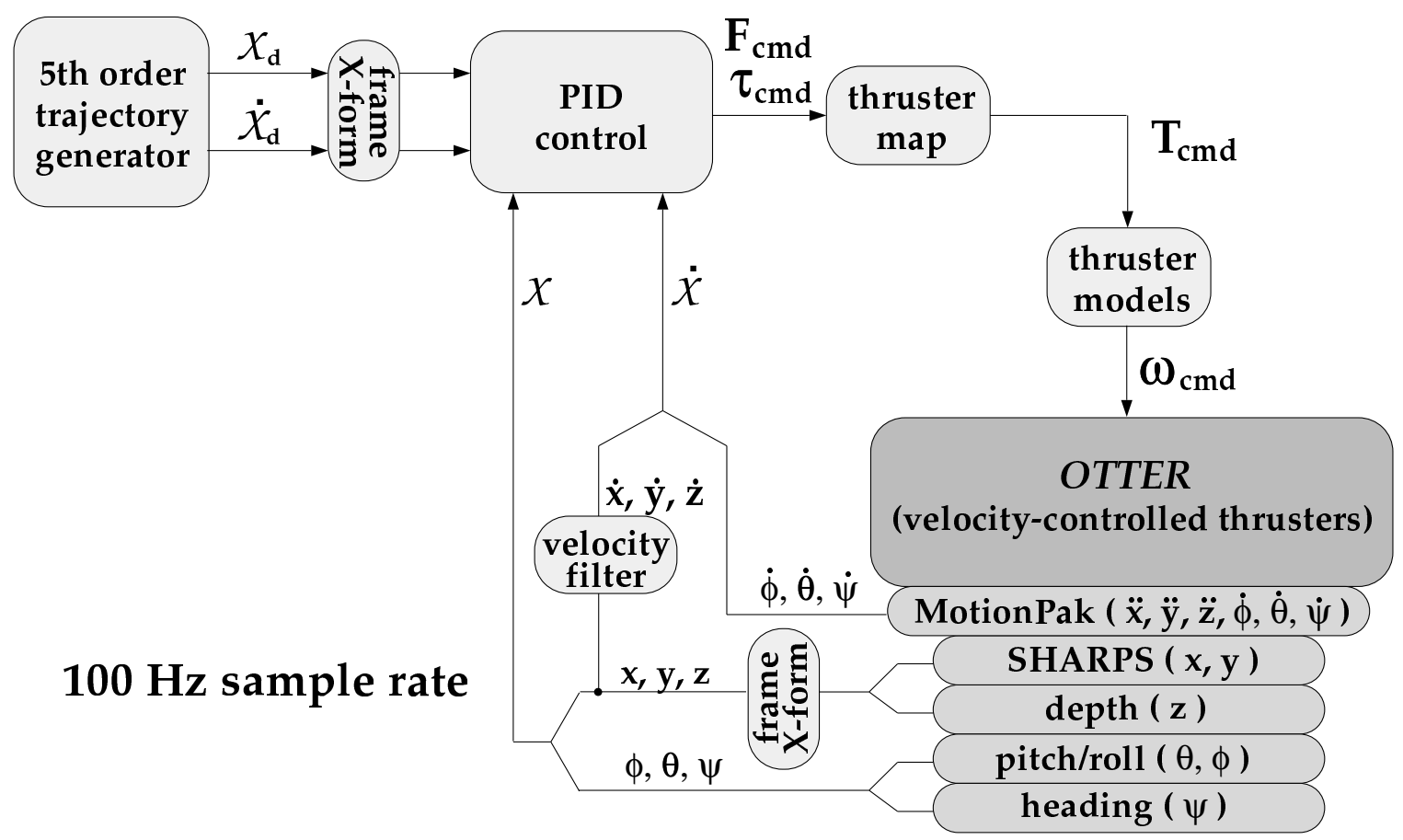

Figure 7: Vehicle Feedback Control Block Diagram

This schematic shows the vehicle feedback control block diagram. The controller is implemented digitally and runs at $100 \mathrm{~Hz}$. SHARPS position measurements are updated at $2.5 \mathrm{~Hz}$ while the heading (yaw) measurement from the flux-gate compass is updated at $10 \mathrm{~Hz}$. All other sensors are sampled at the full sample rate. Thrusters are velocity controlled using their internal electronics. 


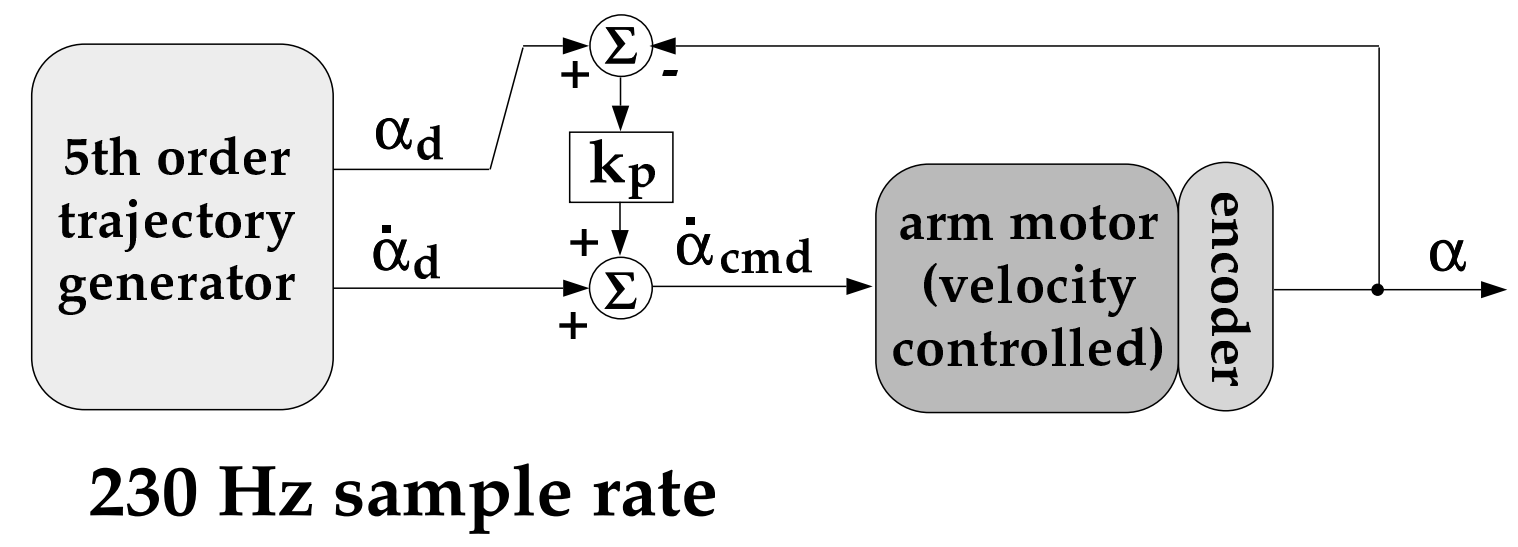

Figure 8: Arm Feedback Control Block Diagram

This schematic shows the arm feedback control block diagram. The controller is implemented digitally at $230 \mathrm{~Hz}$. The arm motor was velocity controlled by a $1 \mathrm{kHz}$, high-gain feedback controller implemented on the $68 \mathrm{HC11}$ microcontroller that is part of the motor commutation electronics. 


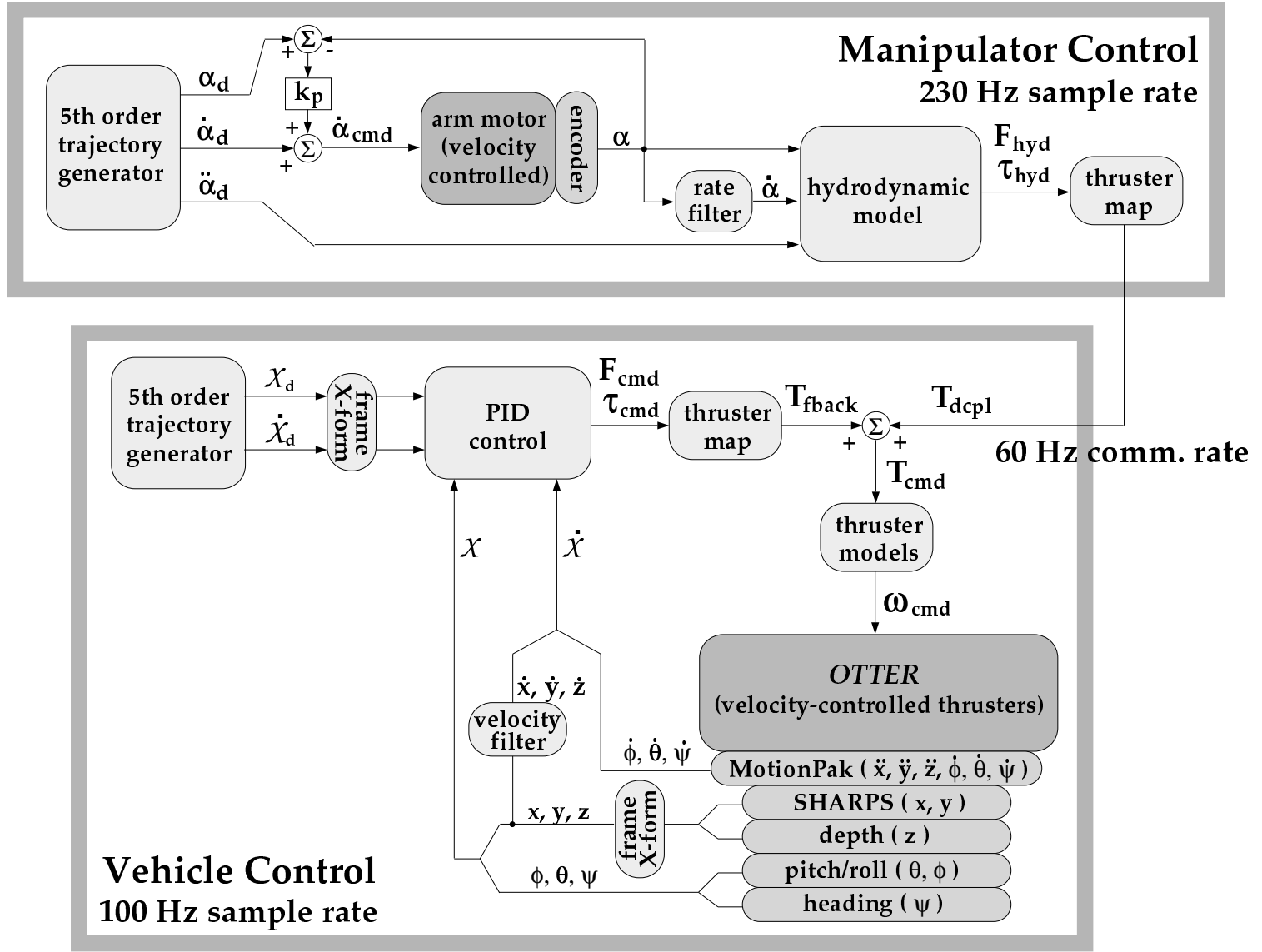

Figure 9: Coordinated-Control Implementation Block Diagram

This schematic shows the block diagram of the coordinated-control scheme as implemented on the OTTER vehicle. The arm controller runs in one VME cage at $230 \mathrm{~Hz}$, while the vehicle controller runs in a second VME cage at $100 \mathrm{~Hz}$. The hydrodynamic model information is sent from the arm controller to the vehicle controller over an Ethernet connection at $60 \mathrm{~Hz}$. 


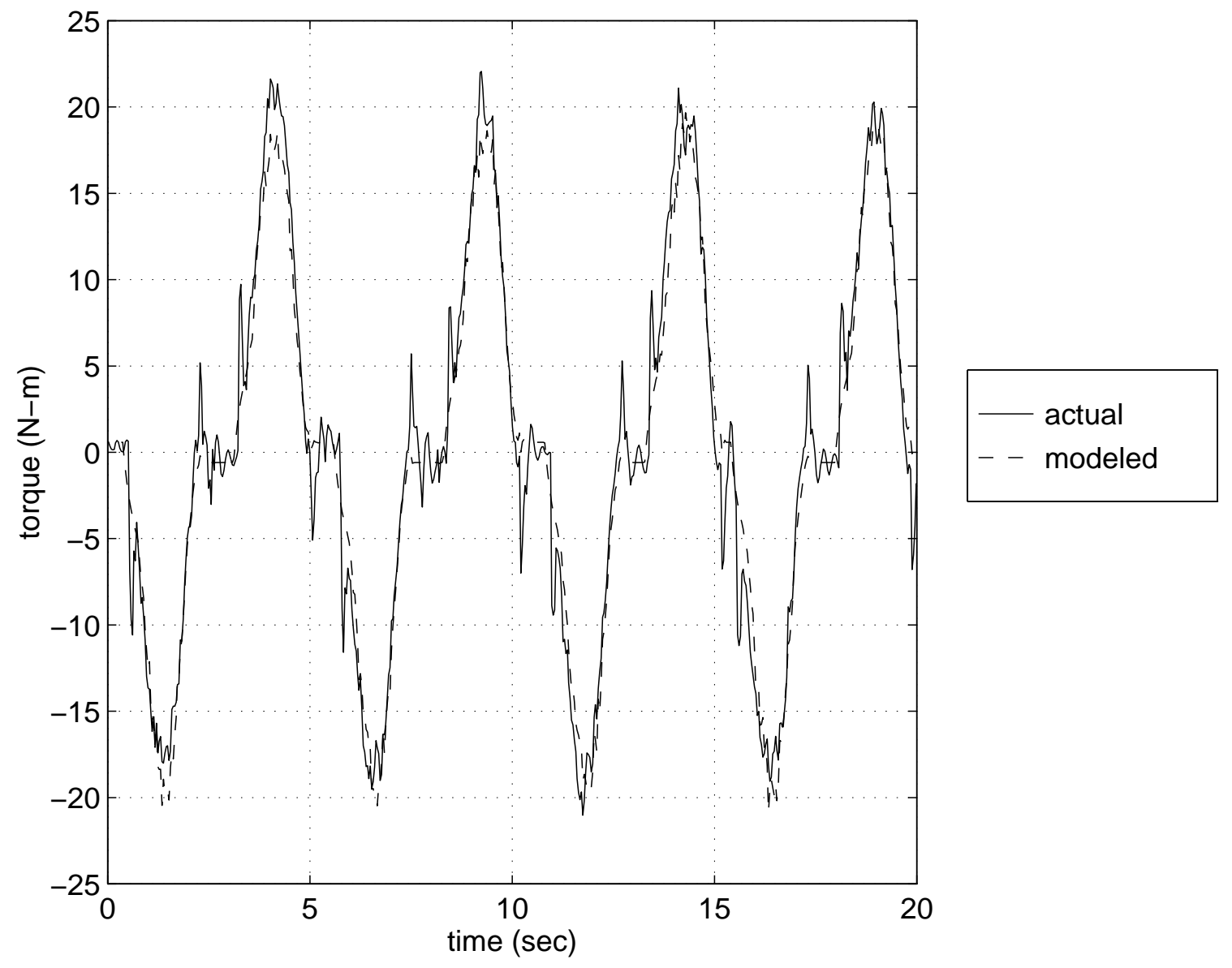

Figure 10: Measured and Modeled Joint Torque on OTTER Vehicle.

This plot compares measured joint torque with the modeled joint torque when the arm was moved back and forth on the OTTER vehicle. 


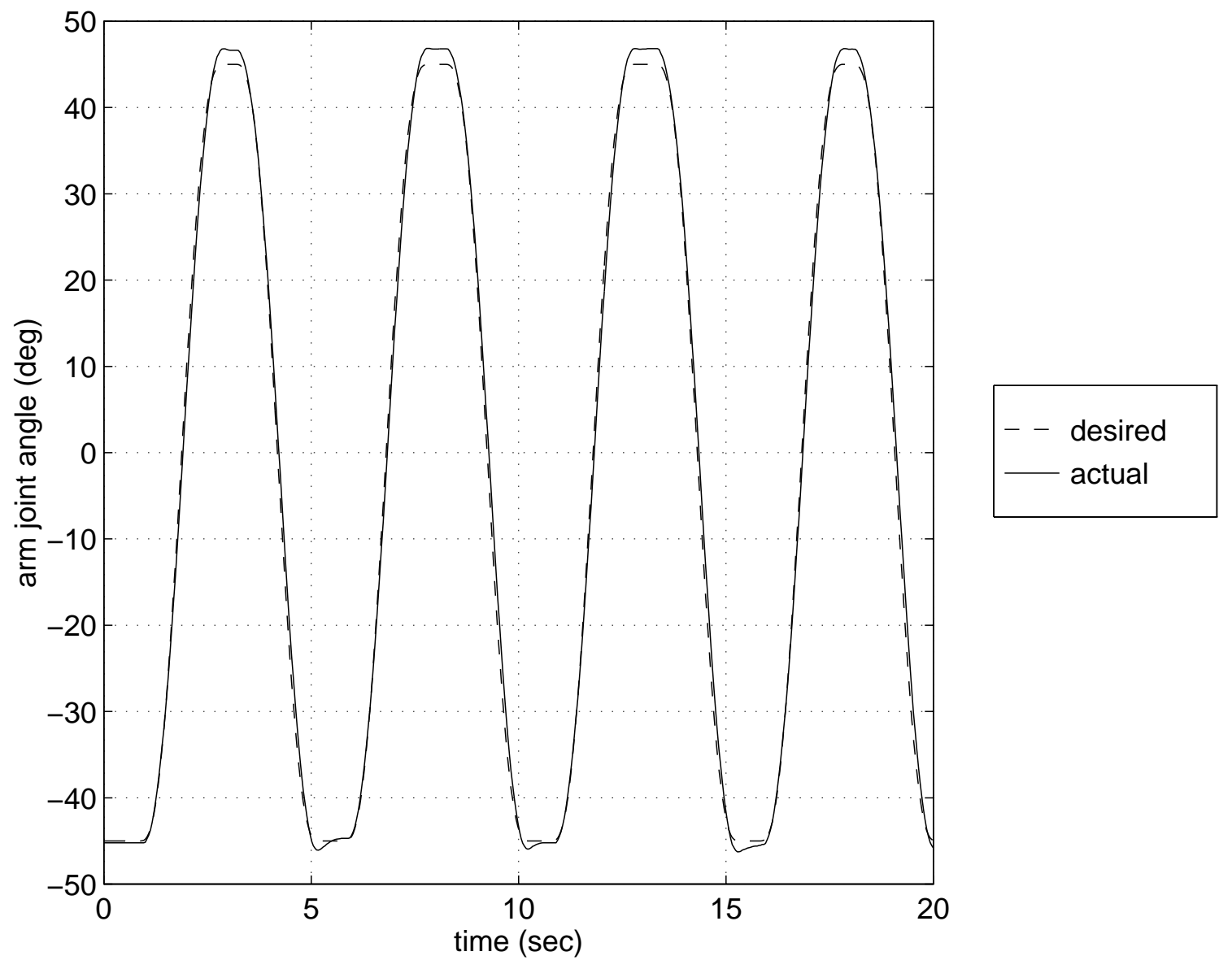

Figure 11: Arm-Joint-Angle Time History

This figure shows the arm joint angle versus time for the multiple-swing arm motion results shown in Figures 12 through 18. 


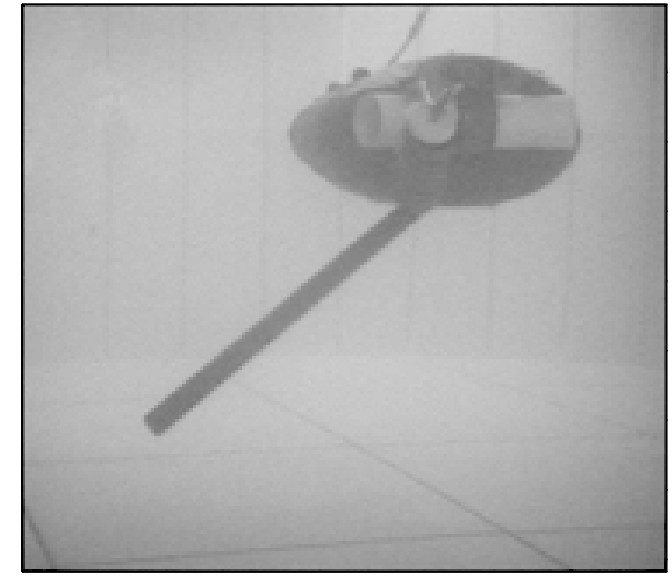

$\# 1:$ time $=0.0 \mathrm{sec}$

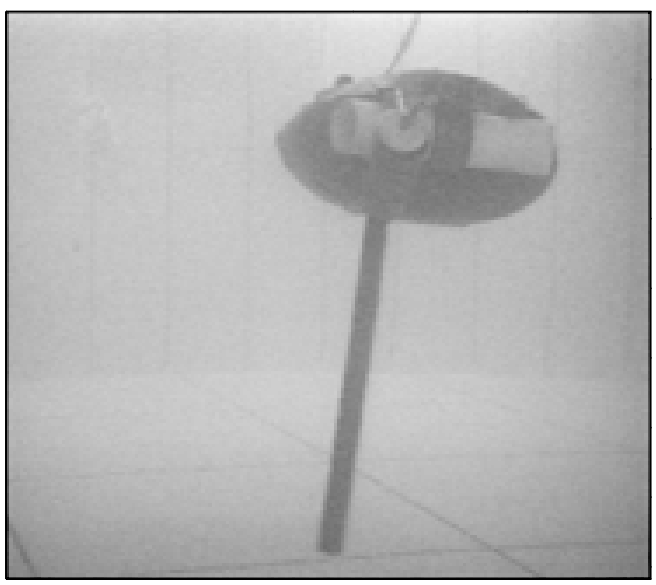

$\# 2:$ time $=0.93 \mathrm{sec}$

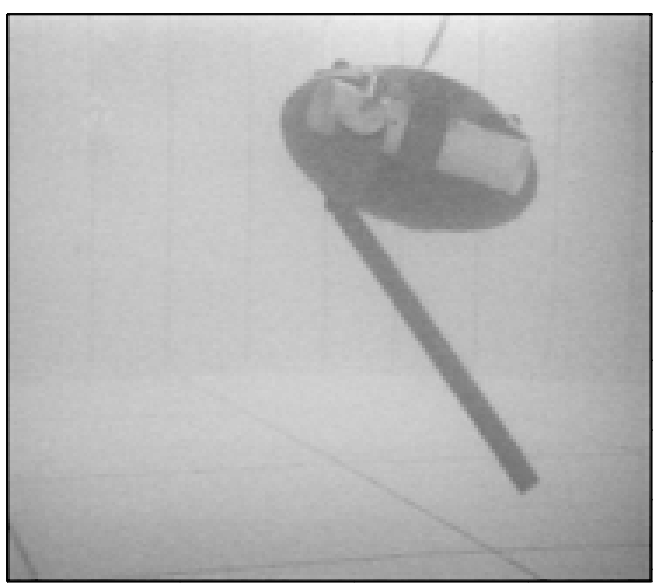

\#3: time $=2.07 \mathrm{sec}$

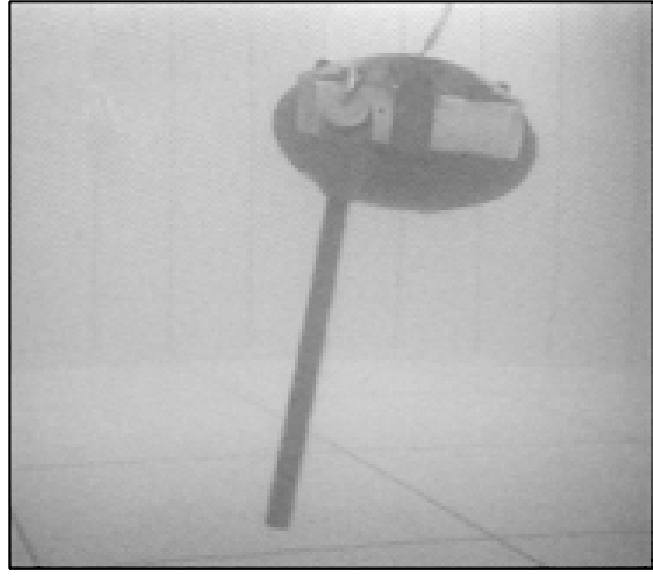

\#4: time $=3.07 \mathrm{sec}$

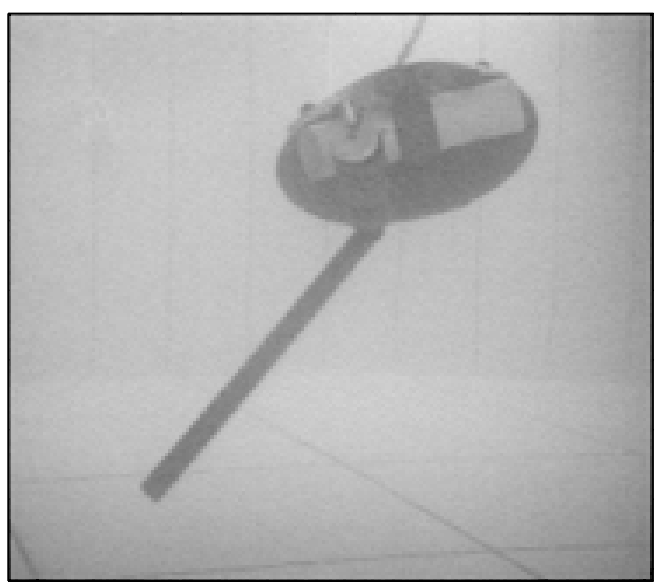

$\# 5:$ time $=4.03 \mathrm{sec}$

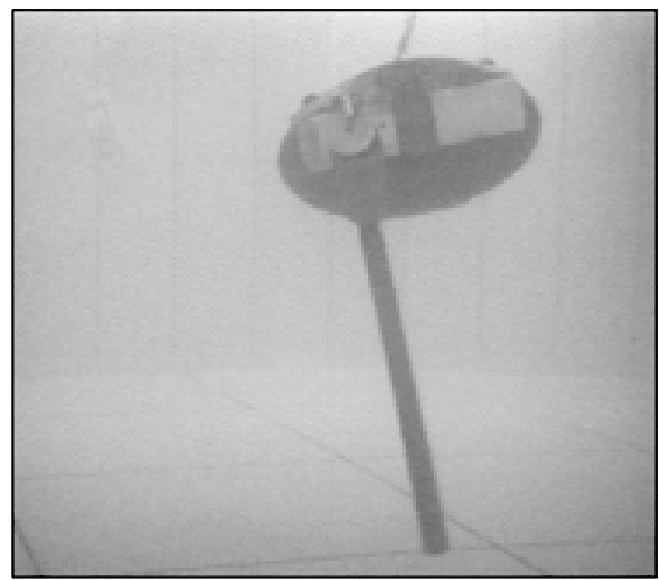

\#6: time $=5.33 \mathrm{sec}$

\section{Figure 12: Arm/Vehicle Response - No Vehicle Control}

For this sequence, all control commands to the thrusters were disabled. The vehicle rolled as much as 18 degrees in both directions from its nominal horizontal position. In yaw, the vehicle rotated about 15 degrees from its initial heading angle. 


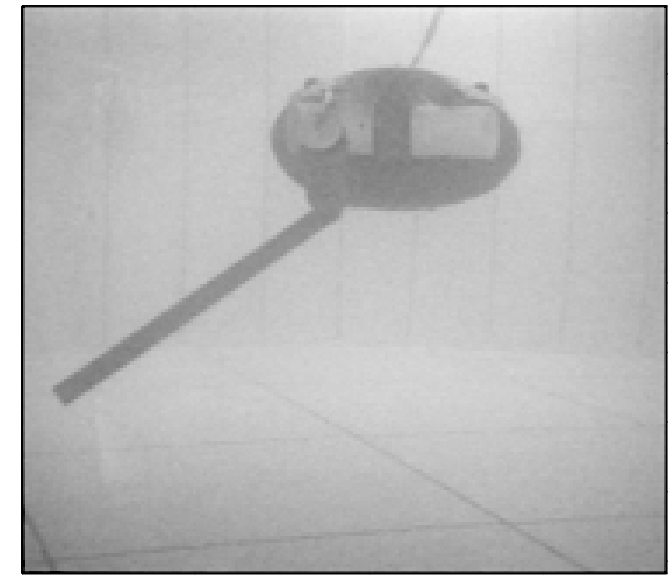

$\# 1:$ time $=0.0 \mathrm{sec}$

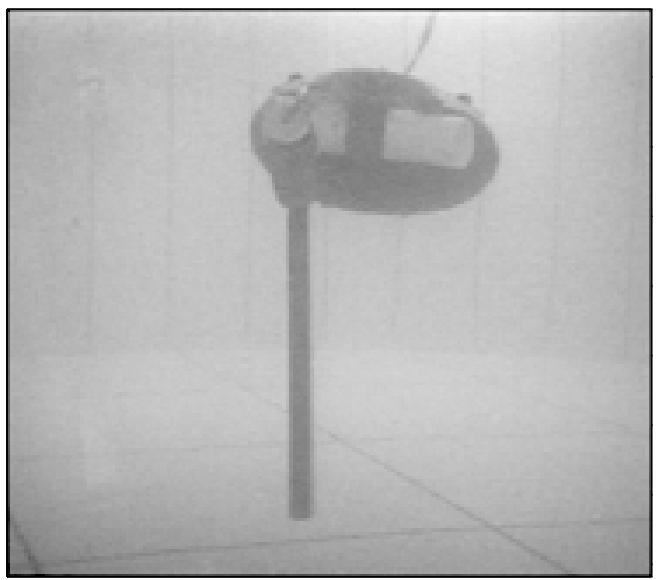

\#2: time $=1.07 \mathrm{sec}$

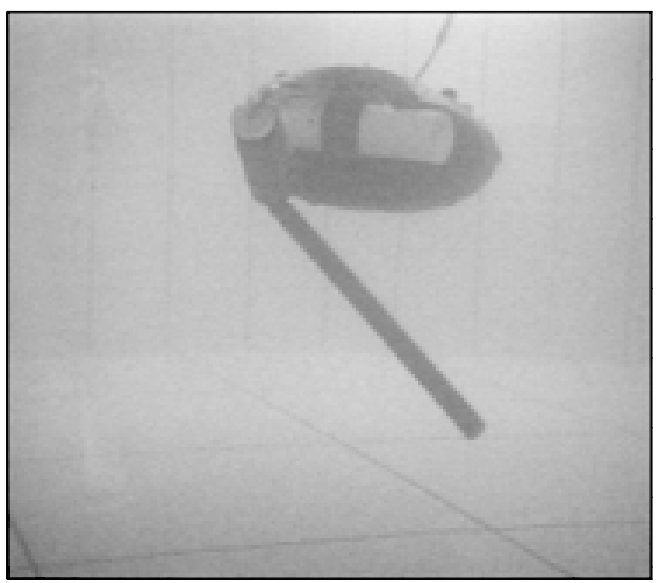

\#3: time $=2.20 \mathrm{sec}$

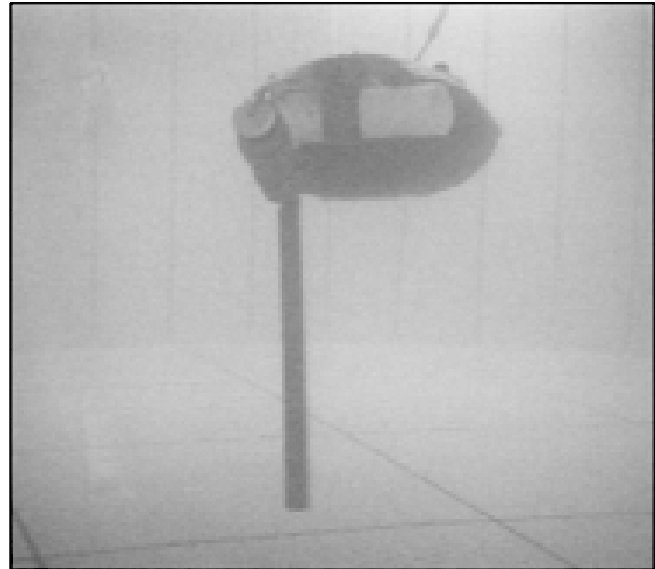

\#4: time $=3.17 \mathrm{sec}$

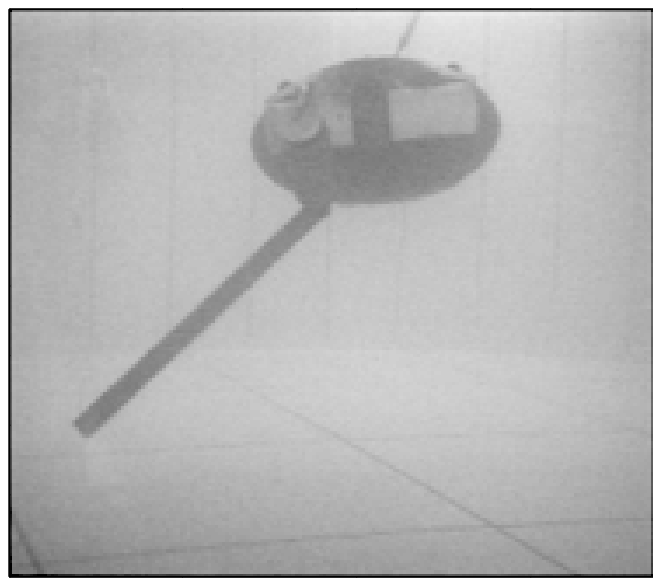

$\# 5:$ time $=4.33 \mathrm{sec}$

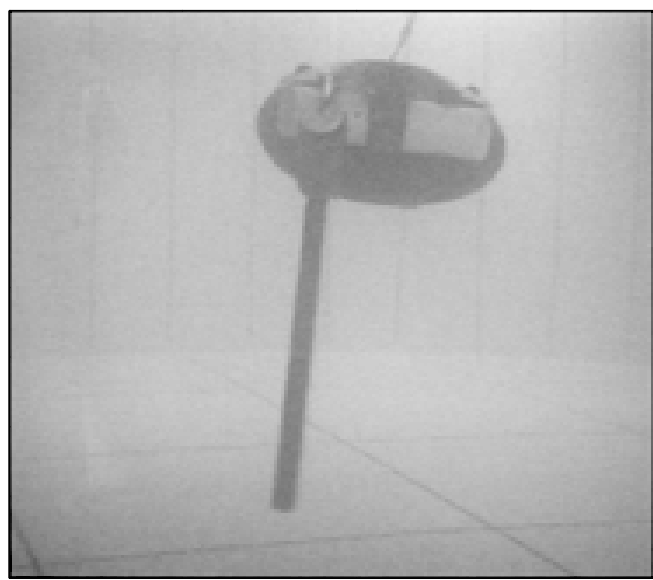

\#6: time $=5.40 \mathrm{sec}$

\section{Figure 13: Arm/Vehicle Response — Feedback Control Only}

In this sequence, the vehicle was under position and attitude feedback control. Roll errors were as large as 9 degrees in both directions, while yaw errors were as large as 8 degrees. 


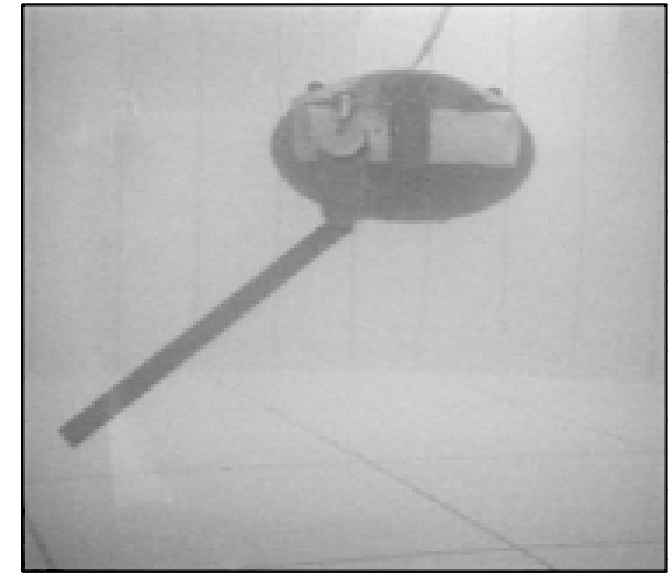

$\# 1:$ time $=0.0 \mathrm{sec}$

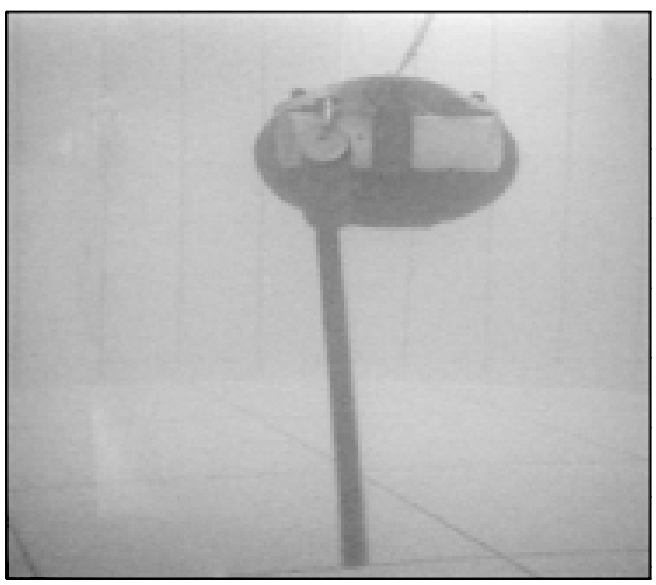

$\# 2:$ time $=1.00 \mathrm{sec}$

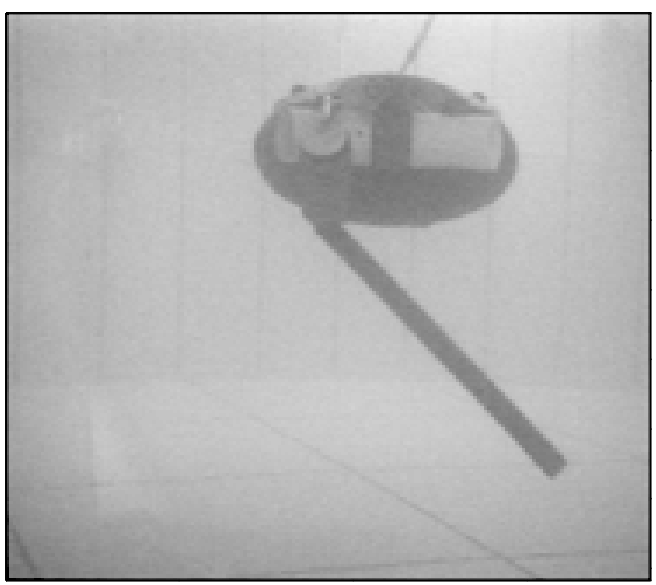

\#3: time $=2.17 \mathrm{sec}$

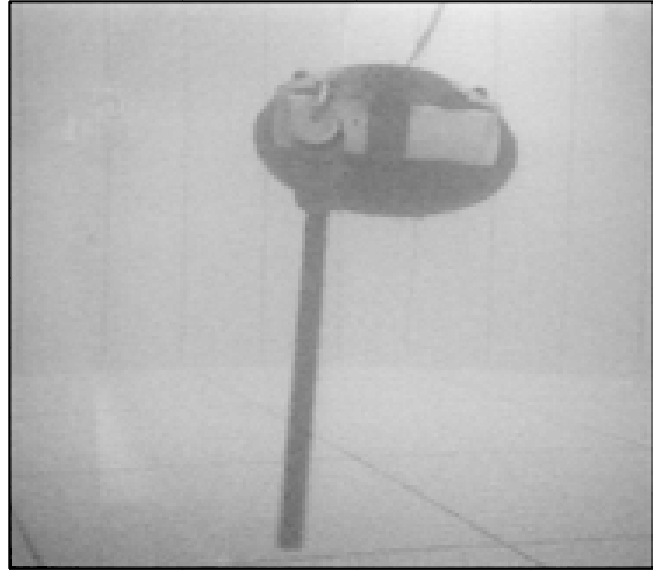

\#4: time $=3.33 \mathrm{sec}$

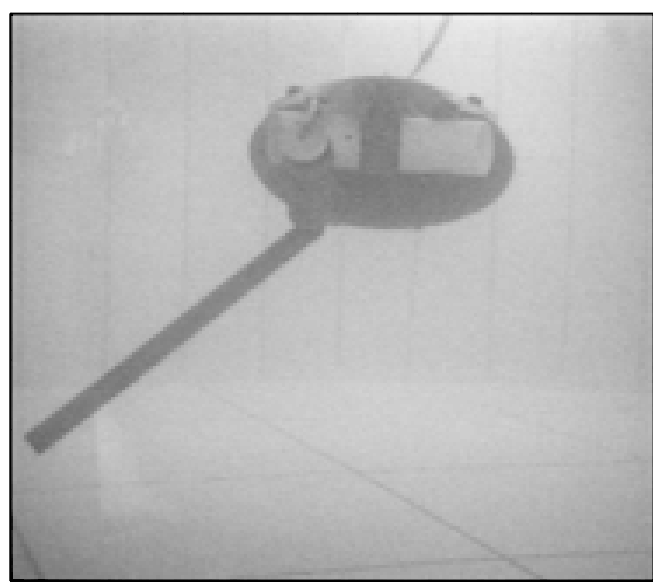

\#5: time $=4.47 \mathrm{sec}$

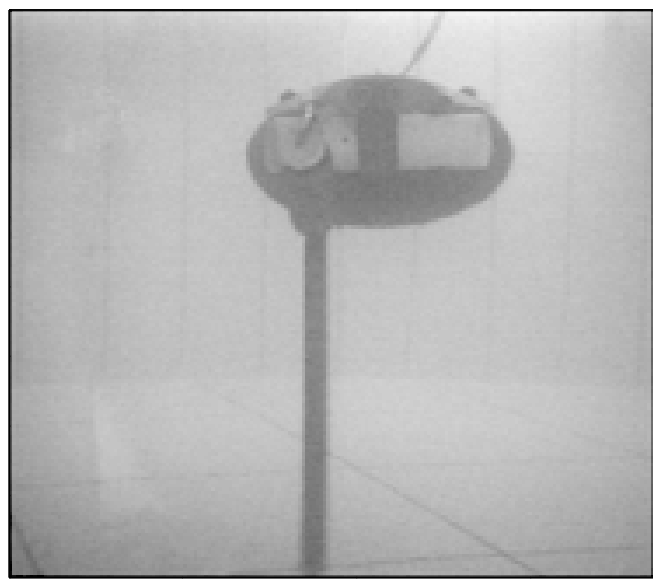

\#6: time $=5.53 \mathrm{sec}$

Figure 14: Arm/Vehicle Response - Decoupling Control Only

For this sequence, vehicle feedback control was disabled, decoupling control was activated. Roll errors were less than 3 degrees. Yaw errors were kept below 4 degrees. Vehicle position drifted slightly due to lack of feedback. 


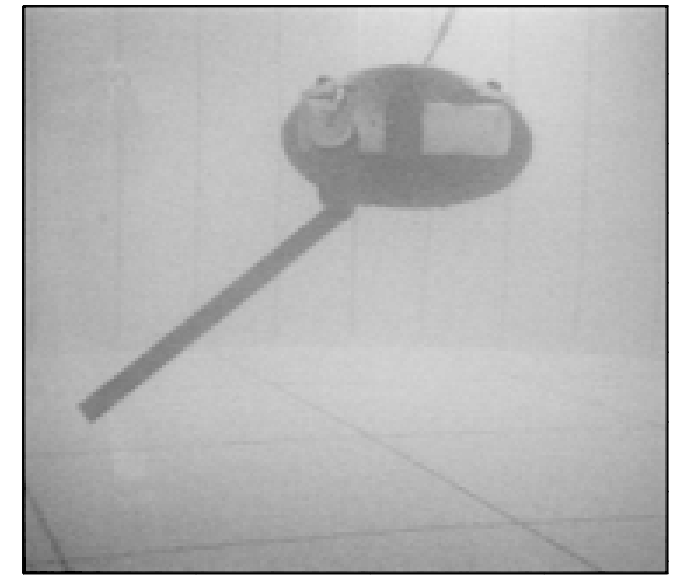

$\# 1:$ time $=0.0 \mathrm{sec}$

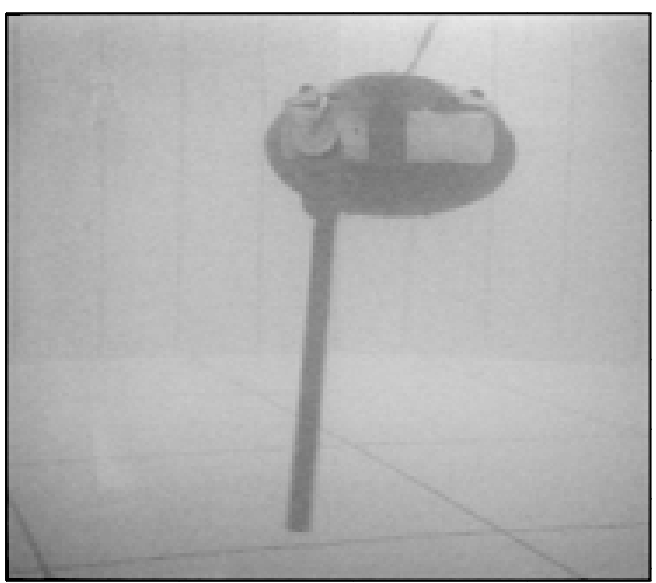

\#2: time $=0.93 \mathrm{sec}$

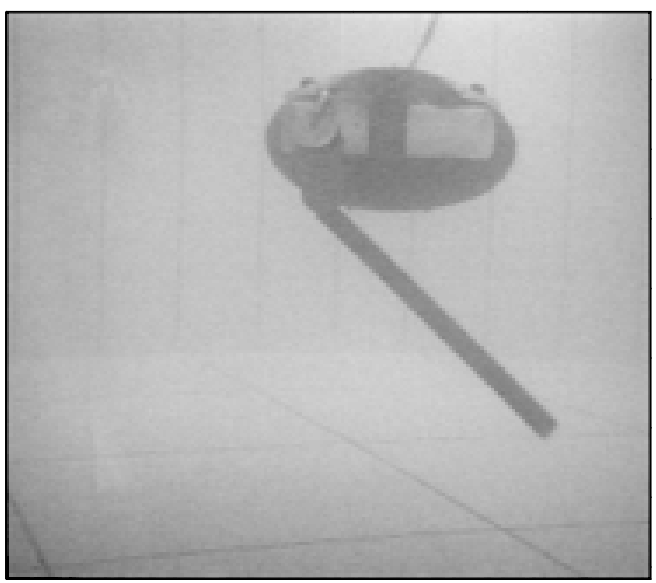

\#3: time $=2.03 \mathrm{sec}$

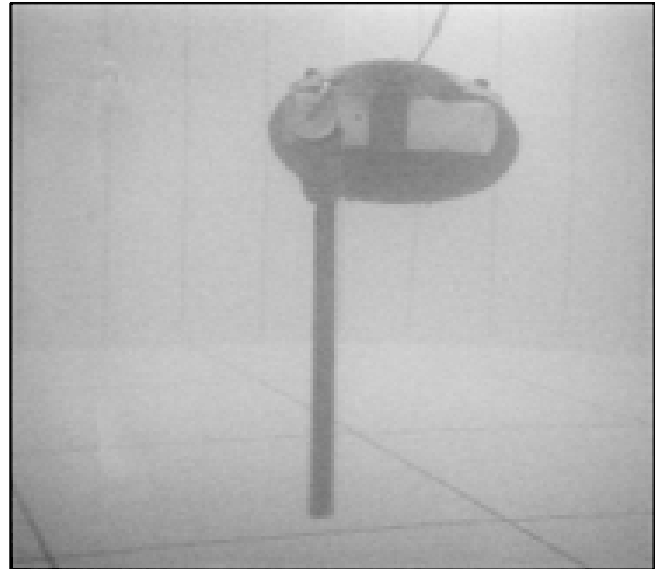

\#4: time $=3.06 \mathrm{sec}$

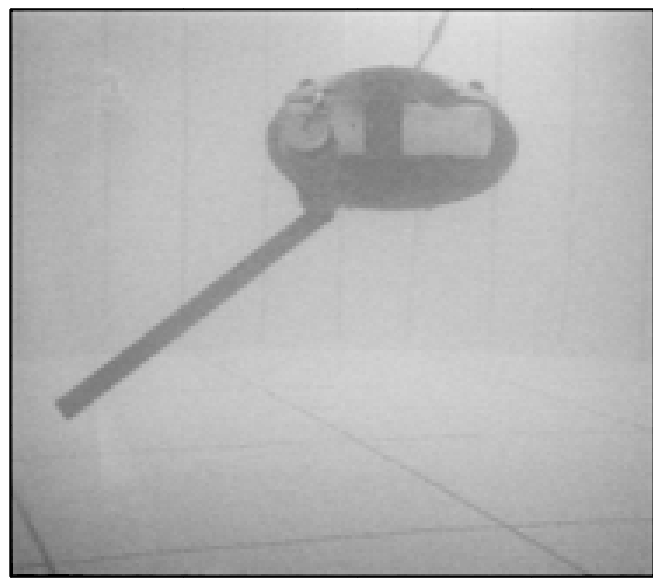

\#5: time $=4.06 \mathrm{sec}$

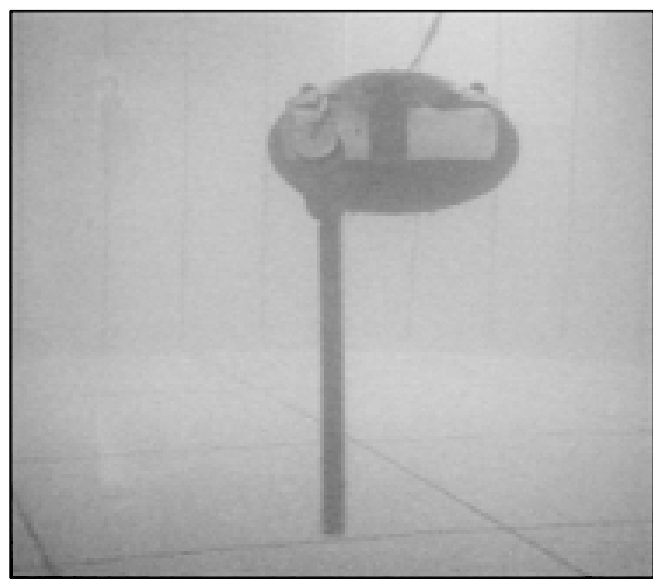

\#6: time $=5.06 \mathrm{sec}$

Figure 15: Arm/Vehicle Response — Feedback with Decoupling Control

In this sequence, both decoupling and feedback control were activated. Roll errors were held below 1.5 degrees. Yaw errors were less than 3 degrees. 

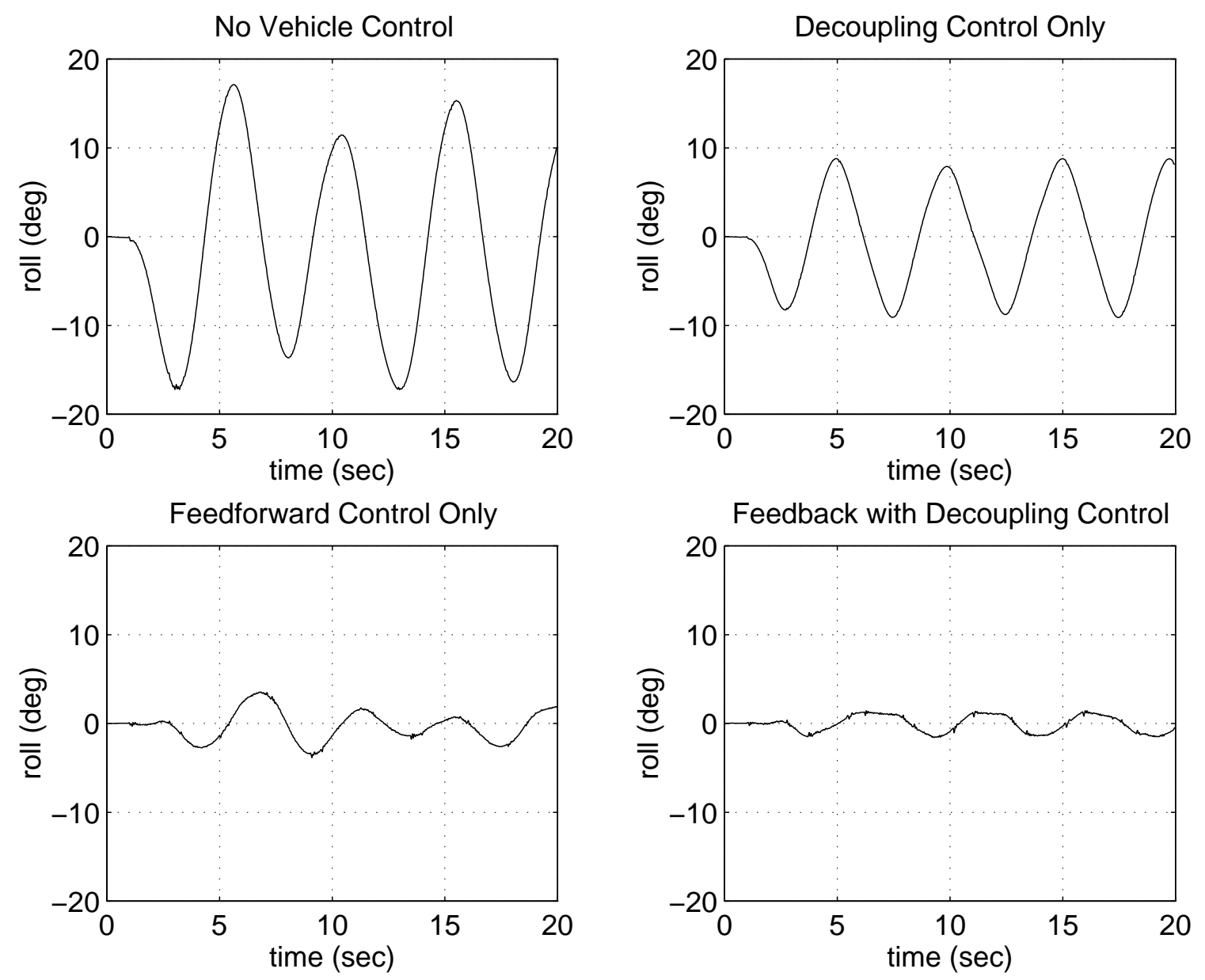

Figure 16: Vehicle Roll Error versus Time

Vehicle roll error is plotted for each controller type. The corresponding arm motion is shown in Figure 11. In each case, the roll mode of the vehicle was excited by the arm motion. The effects of this excitation were reduced significantly by the decoupling control. 

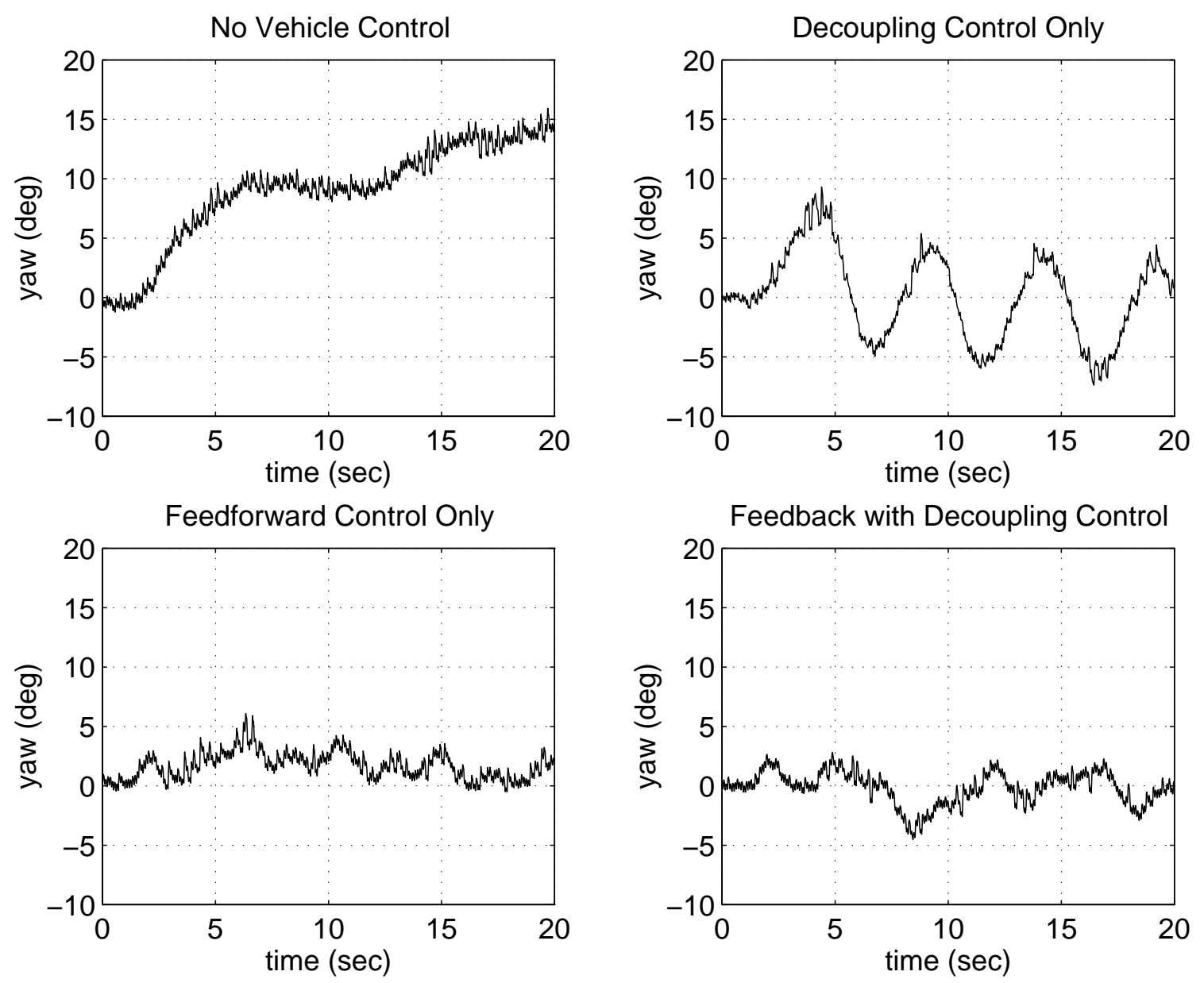

Figure 17: Vehicle Yaw Error versus Time

This figure shows yaw error for each controller type tested. The corresponding arm motion is shown in Figure 11. Yaw errors were roughly three times smaller for the Decoupling Only and Feedback with Decoupling cases than for the Feedback Only case. 

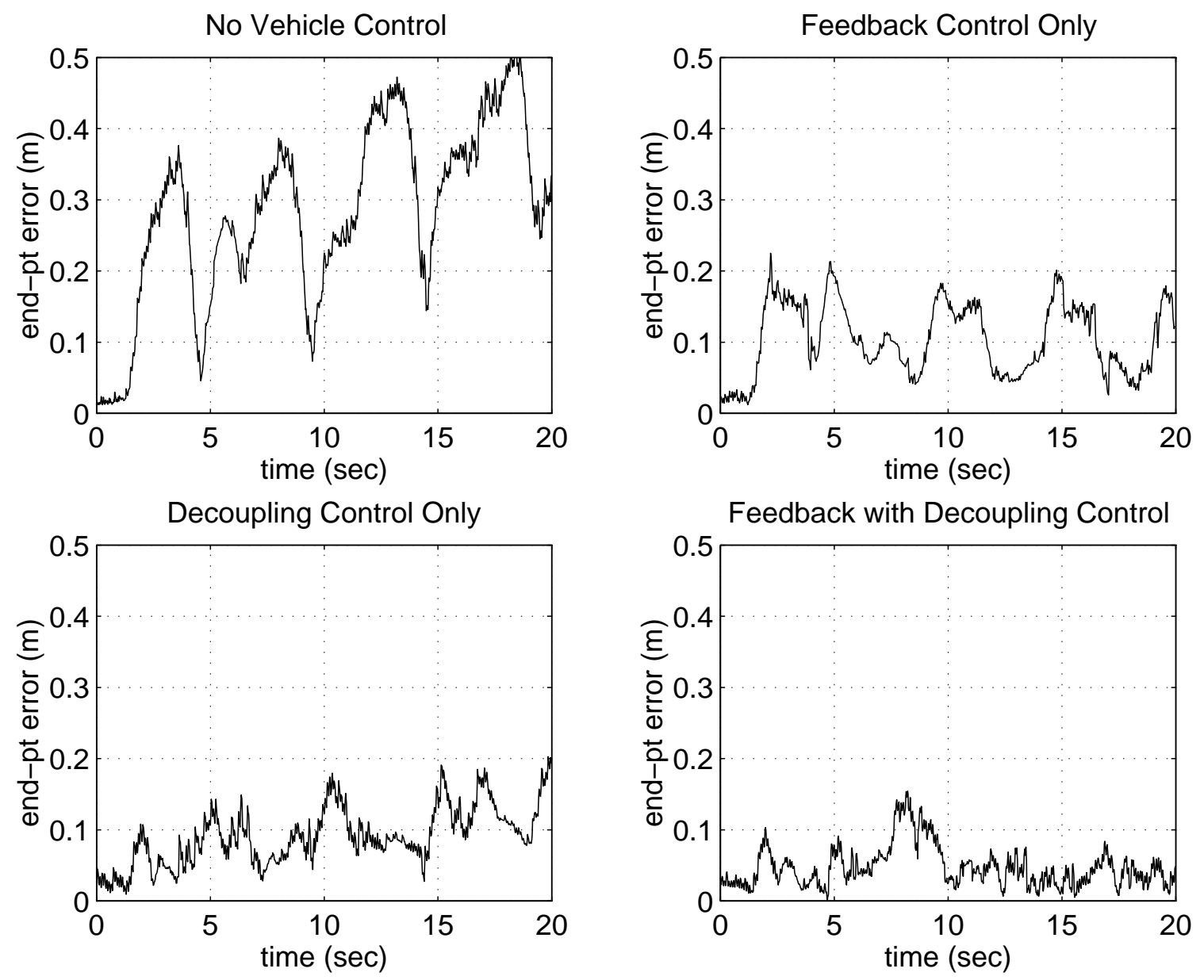

Figure 18: Arm End-Point Error — Multiple Swings

This plot shows a time history of the arm end-point errors corresponding to the arm motions shown in

Figure 11. Mean errors were as follows: No Vehicle Control-28 cm, Feedback Only-11 cm, Decoupling Only-9.1 cm, Feedback with Decoupling-4.6 cm. 

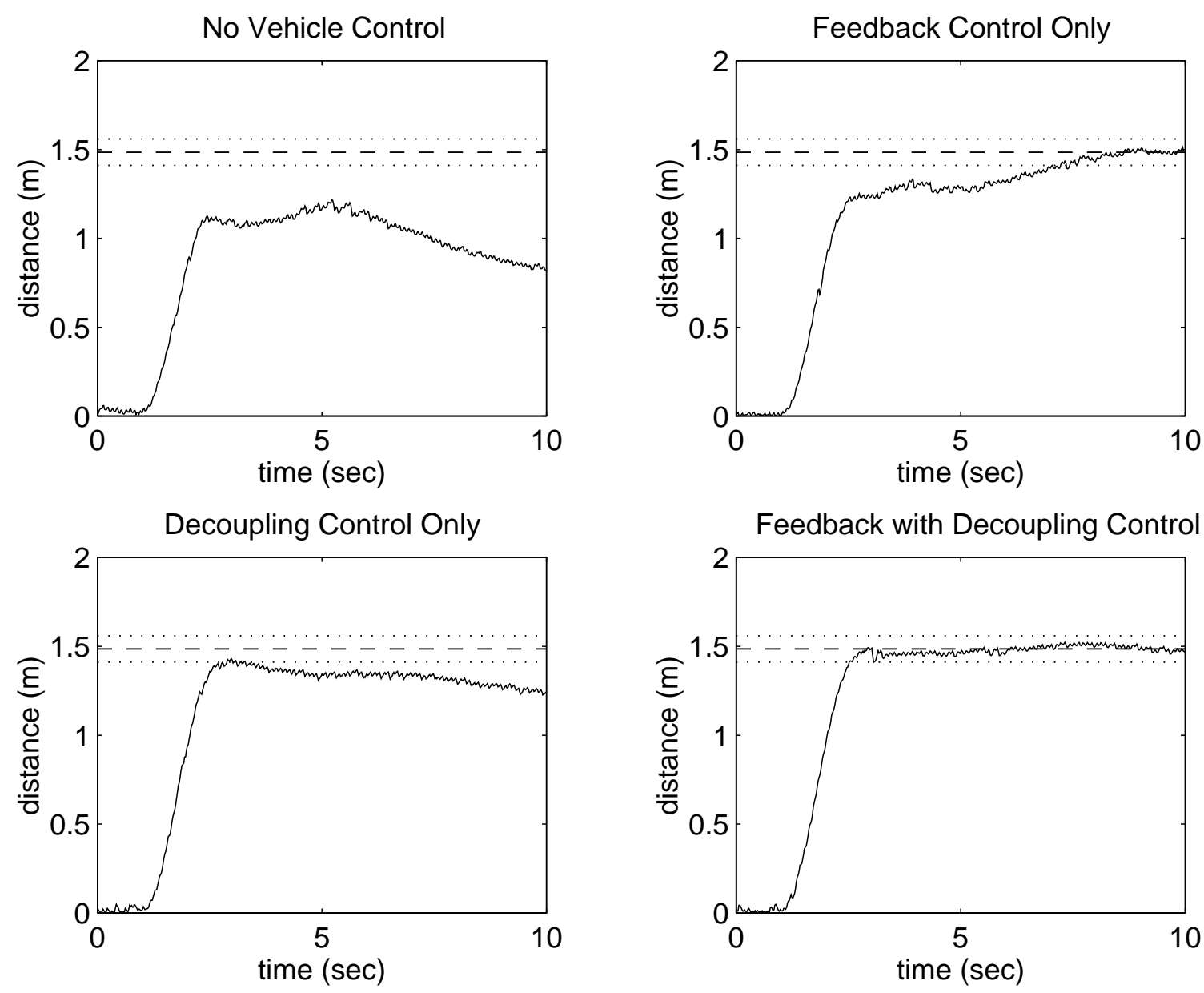

Figure 19: Arm End-Point Settling Time

This figure gives an indication of the settling-time performance of the different controllers. Note that without feedback control, the end point doesn't come within $\pm 5 \%$ of the target. Settling times are approximately 6.5 seconds for the Feedback Only case and 2 seconds for the Feedback with Decoupling case. 

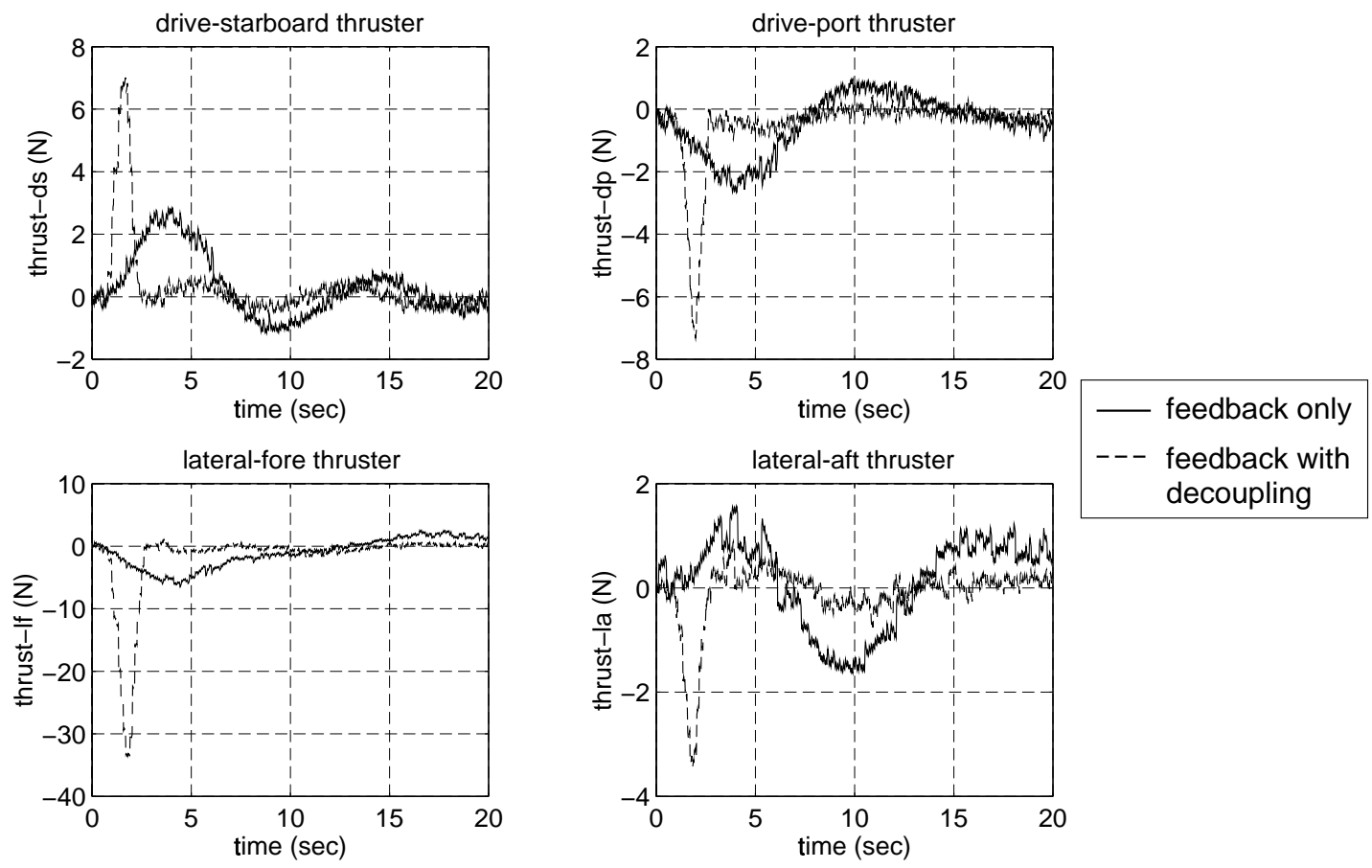

Figure 20: Horizontal Thruster Usage

This figure depicts the thrust commands sent to the individual horizontal thrusters for the motion shown in Figure 19. Thrusts applied in the Feedback with Decoupling case are much larger, but are applied earlier and with much shorter duration. 

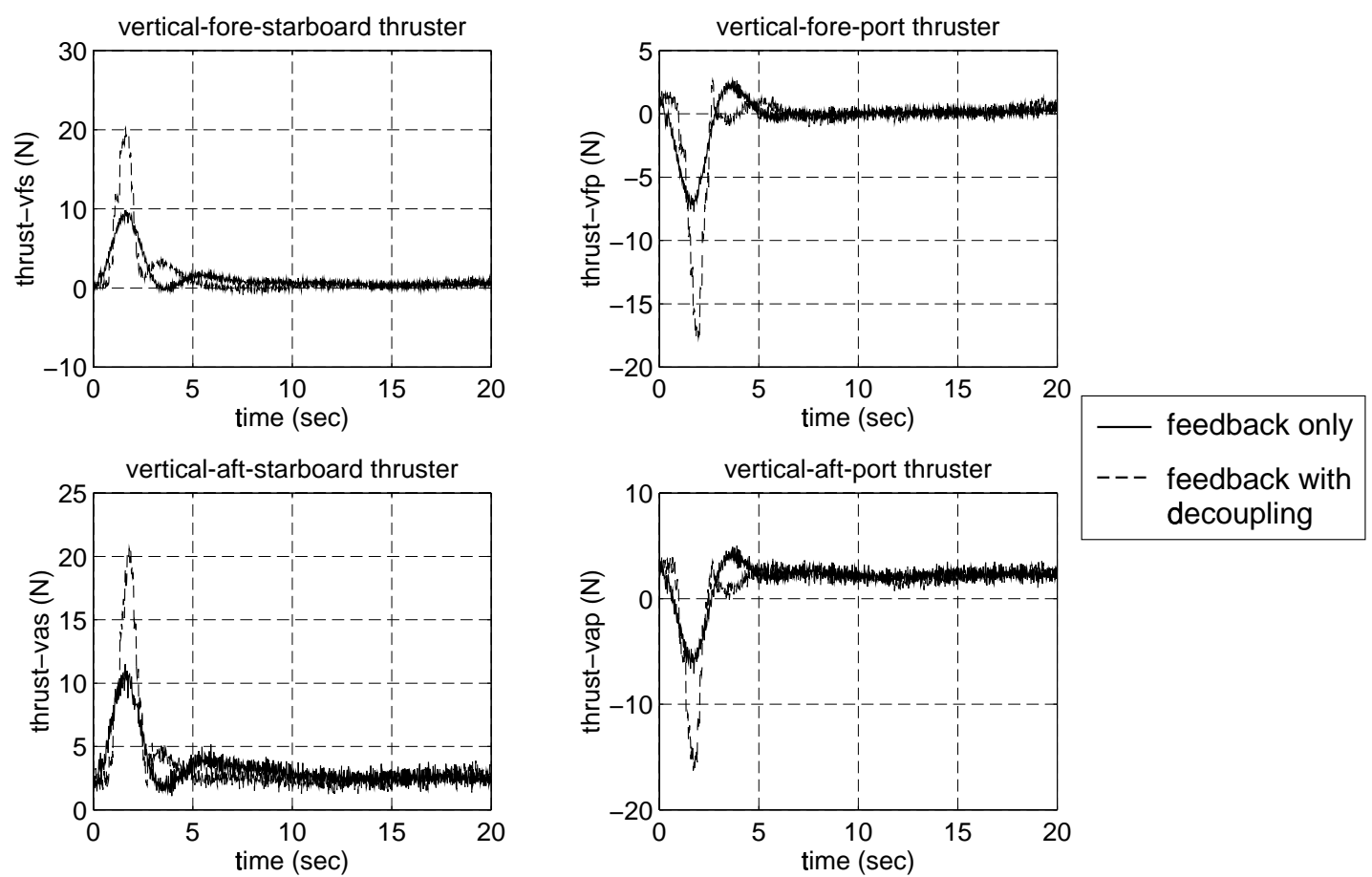

Figure 21: Vertical Thruster Usage

This figure depicts the thrust commands sent to the individual vertical thrusters for the single-swing motion shown in Figure 19. Thrusts applied in the Feedback with Decoupling case are significantly larger during the transient phase. After the transient, both vertical-aft thrusters remain on due to integral control on the pitch error. 


\section{References}

[Anderson, 1992] Jamie M. Anderson. Model development and control of the autonomous benthic explorer. In Proceedings of the Second International Offshore and Polar Engineering Conference, pages 468-472. International Society of Offshore and Polar Engineers, June 1992.

[Craig, 1989] John J. Craig. Introduction to Robotics: Mechanics and Control. Addison-Wesley, 2nd edition, 1989.

[Cristi et al., 1990] Roberto Cristi, Fotis A. Papoulias, and Anthony J. Healey. Adaptive sliding mode control of autonomous underwater vehicles in the dive plane. IEEE Journal of Oceanic Engineering, 15(3):152-160, 1990.

[Dougherty et al., 1988] Frank Dougherty, Tom Sherman, Gary Woolweaver, and Gib Lovell. An autonomous underwater vehicle (AUV) flight control system using sliding mode control. In Oceans '88, pages 1265-1270. IEEE, 1988.

[Goheen and Jefferys, 1990] Kevin R. Goheen and E. Richard Jefferys. Multivariable self-tuning autopilots for autonomous and remotely operated underwater vehicles. IEEE Journal of Oceanic Engineering, 15(3):144-151, 1990.

[Healey and Lienard, 1993] Anthony J. Healey and David Lienard. Multivariable sliding-mode control for autonomous diving and steering of unmanned underwater vehicles. IEEE Journal of Oceanic Engineering, 18(3):327$339,1993$.

[Healey et al., 1995] A. J. Healey, S. M. Rock, S. Cody, D. Miles, and J. P. Brown. Toward an improved understanding of thruster dynamics underwater vehicles. IEEE Journal of Oceanic Engineering, 20(4):354-361, 1995. Accepted for publication in the October 1995 issue.

[Koval, 1994] Elena V. Koval. Automatic stabilization system of underwater manipulation robot. In Oceans '94 Proceedings, pages I-807-I-812. IEEE, September 1994.

[Lévesque and Richard, 1994] Benoît Lévesque and Marc J. Richard. Dynamic analysis of a manipulator in a fluid environment. International Journal of Robotics Research, 13(3):221-231, 1994.

[Mahesh et al., 1991] H. Mahesh, J. Yuh, and R. Lakshmi. A coordinated control of an underwater vehicle and robotic manipulator. The Journal of Robotic Systems, 8(3):339-370, 1991.

[McLain, 1995] Timothy W. McLain. Modeling of Underwater Manipulator Hydrodynamics with Application to the Coordinated Control of an Arm/Vehicle System. PhD thesis, Stanford University, August 1995. Also published as SUDAAR 670.

[McMillan et al., 1994] Scott McMillan, David E. Orin, and Robert B. McGhee. Efficient dynamic simulation of an unmanned underwater vehicle with a manipulator. In Proceedings of the International Conference on Robotics and Automation, pages 1133-1140, 1994. 
[Rea, 1992] Real-Time Innovations, Inc., 155A Moffet Park Drive, Suite 111, Sunnyvale, CA 94089. ControlShell User's Manual: A Real-time Software Framework, 4.2a edition, November 1992.

[Sarpkaya and Garrison, 1963] Turgut Sarpkaya and C. J. Garrison. Vortex formation and resistance in unsteady flow. Journal of Applied Mechanics, pages 16-24, March 1963.

[Sarpkaya, 1963] Turgut Sarpkaya. Lift, drag, and added-mass coefficients for a circular cylinder immersed in a time-dependent flow. Journal of Applied Mechanics, pages 13-15, March 1963.

[Tarn et al., 1995] T.J. Tarn, G.A. Shoults, and S. Yang. Dynamical model for a free-floating underwater robotic vehicle with an n-axis manipulator. In Proceedings of the U.S.-Portugal Workshop on Undersea Robotics and Intelligent Control, Lisboa, March 1995.

[Wang et al., 1993] H. H. Wang, R. L. Marks, S. M. Rock, and M. J. Lee. Task-Based Control Architecture for an Untethered, Unmanned Submersible. In Proceedings of the 8th Annual Symposium of Unmanned Untethered Submersible Technology, pages 137-147. Marine Systems Engineering Laboratory, Northeastern University, September 1993.

[Wang et al., 1996] Howard Wang, Stephen M. Rock, and Michael J. Lee. OTTER: The design and development of an intelligent underwater robot. Journal of Autonomous Robots, 3:299-322, 1996.

[Win, 1993] Wind River Systems, Inc., 1010 Atlantic Avenue, Alameda, CA 94501-1147. VxWorks Programmer's Guide 5.1, December 1993.

[Yoerger and Slotine, 1985] Dana R. Yoerger and Jean-Jaques E. Slotine. Robust trajectory control of underwater vehicles. IEEE Journal of Oceanic Engineering, 10(4):462-470, 1985.

[Yoerger and Slotine, 1991] Dana R. Yoerger and Jean-Jaques E. Slotine. Adaptive sliding control of an experimental underwater vehicle. In Proceedings of the International Conference on Robotics and Automation, pages 2746-2751. IEEE, April 1991.

[Yuh, 1990] Junku Yuh. A neural net controller for underwater robotic vehicles. IEEE Journal of Oceanic Engineering, 15(3):161-166, 1990.

\section{Authors}

Timothy W. McLain received B.S. degree in 1986 and M.S. degree in 1987 in Mechanical Engineering from Brigham Young University. From 1987 to 1989, he worked at the Center for Engineering Design at the University of Utah, where he was involved in modeling and simulation of hydraulic and electromechanical actuation systems, actuator and control system development, and force transducer design. From 1989 through 1995, Dr. McLain was a Research Assistant in the Aerospace Robotics Laboratory at Stanford University. His Ph.D. research, which was done 
in cooperation with the Monterey Bay Aquarium Research Institute, focused on the hydrodynamics of underwater manipulators and the design of control systems for underwater vehicles and manipulators. Currently, Dr. McLain is an Assistant Professor of Mechanical Engineering at Brigham Young University where his research interests center on the control of hydraulic and pneumatic actuation systems.

Stephen M. Rock received his S.B. and S.M. degrees in Mechanical Engineering from the Massachusetts Institute of Technology in 1972, and the Ph.D. degree in Applied Mechanics from Stanford University in 1978. Dr. Rock joined the Stanford faculty in 1988, and is now an Associate Professor in the department of Aeronautics and Astronautics. Prior to joining the Stanford faculty, Dr. Rock led the Controls and Instrumentation Department of Systems Control Technology, Inc. In his eleven years at SCT, he performed and led research in integrated control; fault detection, isolation and accommodation; turbine engine modeling and control; and parameter identification. Dr. Rock's current research interests include the development and experimental validation of control approaches for robotic systems and for vehicle applications. A major focus is both the high-level and low-level control of underwater robotic vehicles.

Michael J. Lee received his S.B. degree in Electrical Engineering form the Massachusetts Institute of Technology in 1975 and the M.S. degree in Electrical Engineering from Stanford University in 1979. Mr. Lee joined the Hewlett Packard Co. in 1976 and held several engineering and management positions including Department Manager of the Control Systems Department at HP Laboratories. In 1987, Mr. Lee joined MBARI as one of its founders with the position of Technical Director. Mr. Lee was an Adjunct Professor in Electrical Engineering at Boise State University from 1977 through 1978, and is currently a Consulting Associate Professor at Stanford University. 